\title{
1 Comprehensive Epitope Mapping of Broad Sarbecovirus Neutralizing Antibodies
}

2 Yunlong $\mathrm{Cao}^{1,2, \#, *}$, Ayijiang Yisimayi ${ }^{2,3, \#}$, Fanchong Jian ${ }^{2,4, \#}$, Tianhe Xiao ${ }^{2,5, \#}$, Weiliang 3 Song ${ }^{2,3, \#}$, Jing Wang ${ }^{2,3, \#}$, Shuo Du ${ }^{3, \#}$, Zhiying Zhang ${ }^{3, \#}$, Pulan Liu ${ }^{3, \#}$, Xiaohua Hao ${ }^{6, \#,}$

4 Qianqian $\mathrm{Li}^{7}, \#$, Peng Wang ${ }^{1}$, Ran $\mathrm{An}^{2}$, Yao Wang ${ }^{1}$, Jing Wang ${ }^{1}$, Haiyan Sun ${ }^{1}$, Lijuan 5 Zhao ${ }^{1}$, Wen Zhang ${ }^{6}$, Dong Zhao ${ }^{6}$, Jiang Zheng ${ }^{1}$, Lingling $\mathrm{Yu}^{1}$, Can $\mathrm{Li}^{1}$, Na Zhang ${ }^{1}$, Rui

6 Wang ${ }^{1}$, Xiao $\mathrm{Niu}^{2}$, Sijie Yang ${ }^{2,8}$, Xuetao Song ${ }^{1}$, Linlin Zheng ${ }^{1}$, Zhiqiang $\mathrm{Li}^{8,9}$,

7 Qingqing $\mathrm{Gu}^{1}$, Fei Shao ${ }^{1}$, Weijin Huang ${ }^{7}$, Youchun Wang ${ }^{7}$, Ronghua Jin ${ }^{6, *}$, Junyu

$8 \mathrm{Xiao}^{3,8,9, *}$, Xiaoliang Sunney $\mathrm{Xie}^{1,2, *}$

${ }^{1}$ Changping Laboratory, Beijing, P.R. China.

${ }^{2}$ Biomedical Pioneering Innovation Center (BIOPIC), Peking University, Beijing, P.R. China.

${ }^{3}$ School of Life Sciences, Peking University, Beijing, P.R. China.

${ }^{4}$ Joint Graduate Program of Peking-Tsinghua-NIBS, Academy for Advanced Interdisciplinary Studies, Peking University, Beijing, China.

${ }^{5}$ College of Chemistry and Molecular Engineering, Peking University, Beijing, P.R. China.

${ }^{6}$ Beijing Ditan Hospital, Capital Medical University, Beijing, P.R. China.

${ }^{7}$ Division of HIV/AIDS and Sex-transmitted Virus Vaccines, Institute for Biological Product Control, National Institutes for Food and Drug Control (NIFDC), Beijing, P.R. China.

${ }^{8}$ Peking-Tsinghua Center for Life Sciences, Peking University, Beijing, P.R. China ${ }^{9}$ Academy for Advanced Interdisciplinary Studies, Peking University, Beijing, P.R. China.

*Correspondence: Yunlong Cao (yunlongcao@pku.edu.cn); Ronghua Jin (ronghuajin@ccmu.edu.cn); Junyu Xiao (junyuxiao@pku.edu.cn); Xiaoliang Sunney Xie (sunneyxie@biopic.pku.edu.cn)

${ }^{\#}$ These authors contributed equally. 


\section{Abstract}

Constantly emerging SARS-CoV-2 variants, such as Omicron BA.1, BA.1.1 and BA.2, pose a severe challenge to COVID-19 control $^{1-10}$. Broad-spectrum antibody therapeutics and vaccines are needed for defending against future SARS-CoV-2 variants and sarbecovirus pandemics ${ }^{11-14}$; however, we have yet to gain a comprehensive understanding of the epitopes capable of inducing broad sarbecovirus neutralization. Here, we report the identification of 241 anti-RBD broad sarbecovirus neutralizing antibodies isolated from 44 SARS-CoV-2 vaccinated SARS convalescents. Neutralizing efficacy of these antibodies against D614G, SARS-CoV-1, Omicron variants (BA.1, BA.1.1, BA.2), RATG13 and Pangolin-GD is tested, and their binding capability to 21 sarbecovirus RBDs is measured. High-throughput yeast-display mutational screening was further applied to determine each antibody's RBD escaping mutation profile, and unsupervised epitope clustering based on escaping mutation hotspots was performed ${ }^{7,15-18}$. A total of 6 clusters of broad sarbecovirus neutralizing antibodies with diverse breadth and epitopes were identified, namely Group E1 (S309 ${ }^{19}$, BD55-3152 site), E3 (S2H97 ${ }^{20}$ site), F1 (CR3022 ${ }^{21}, \mathrm{~S} 304^{22}$ site), F2 (DH104723, BD553500 site), F3 (ADG-2 ${ }^{24}, \mathrm{BD} 55-3372$ site) and B' (S2K146 ${ }^{25}$ site). Members of E1, F2 and F3 demonstrate the highest neutralization potency; yet, Omicron, especially BA.2, has evolved multiple mutations (G339D, N440K, T376A, D405N, R408S) to escape antibodies of these groups. Nevertheless, broad sarbecovirus neutralizing antibodies that survived Omicron would serve as favorable therapeutic candidates. Furthermore, structural analyses of selected drug candidates propose two non-competing antibody pairing strategies, E1-F2 and E1-F3, as broad-spectrum antibody cocktails. Together, our work provides a comprehensive epitope map of broad sarbecovirus neutralizing antibodies and offers critical instructions for designing broad-spectrum vaccines. 
Main

The recent emergence and global spreading of severe acute respiratory syndrome coronavirus 2 (SARS-CoV-2) variant Omicron has posed a critical challenge to the efficacy of COVID-19 vaccines and neutralizing antibody therapeutic ${ }^{2-5}$. Due to multiple mutations to the spike protein and its receptor-binding domain (RBD), Omicron can cause severe neutralizing antibody evasion ${ }^{5-9}$. The continuous evolution of Omicron, such as BA.1.1 and BA.2, may lead to an even stronger immune escape ${ }^{2,8,10}$. For pandemic preparedness, pan-sarbecovirus therapeutics and vaccines that are effective against future SARS-CoV-2 variants and other sarbecoviruses are urgently needed ${ }^{11-14}$. Several broad sarbecovirus neutralizing antibodies have been reported ${ }^{20,23-}$ 34; however, since the number of antibodies studied has been limited, we still lack a comprehensive understanding of the RBD epitopes that could induce broad sarbecovirus neutralizing antibodies.

SARS convalescents who were subsequently vaccinated against SARS-CoV-2 may carry a large number of broad-spectrum neutralizing antibodies ${ }^{19,23,35}$. To study their antibody repertoire, we recruited 44 SARS convalescents and collected their plasma and peripheral blood mononuclear cells (PBMCs) after SARS-CoV-2 vaccination (Supplementary Table 1). The plasma of these individuals exhibited high neutralization ability against both SARS-CoV-1 and SARS-CoV-2 pseudovirus (Extended Data Fig 1a,b). Using flow cytometry, we sorted the memory B cells that could cross-bind to both SARS-CoV-1 and SARS-CoV-2 RBD (Extended Data Fig. 2). Then, we used highthroughput single-cell VDJ sequencing to obtain the B-cell repertoire of these memory B cells ${ }^{36,37}$, and expressed the selected $\operatorname{IgG}$ antibodies in vitro as human $\operatorname{IgG} 1$ monoclonal antibodies. In total, we obtained 1409 SARS-CoV-1 and SARS-CoV-2 RBD binding IgG1 antibodies, where 269 showed SARS-CoV-1 and SARS-CoV-2 cross-neutralizing capability (Supplementary Table 2).

To study the epitope distribution of those bivalent antibodies, we analyzed their escaping mutation profiles using high-throughput yeast display mutant screening that 
covers all possible single residue substitutions in the wildtype RBD background ${ }^{7,15-18}$. We successfully retained the escaping mutation profiles of 241 SARS-CoV-1/SARSCoV-2 cross-neutralizing antibodies as well as 85 non-neutralizing bivalent binding antibodies. Combined with previously characterized antibodies isolated from SARSCoV-2 convalescents and vaccinees ${ }^{36,38,39}$, we collected a library of 715 human SARSCoV-2 IgG1 antibodies with corresponding escaping mutation profiles (Supplementary Data 1). Neutralizing efficacy of these antibodies against sarbecovirus, including D614G, SARS-CoV-1, Omicron variants (BA.1, BA.1.1, BA.2), RATG13 and Pangolin-GD, is tested, and their binding capability to 21 sarbecovirus RBD is measured through ELISA (Supplementary Table 2-3). Based on the escaping mutation profiles, the antibodies could be unsupervised clustered into 10 epitope groups (Fig. 1a, Extended Data Fig. 3, Supplementary Data 2), as described previously7. Group A-D recapitulates our previous antibody clustering ${ }^{7}$, in which the members mainly target the ACE2-binding motif ${ }^{11,22,40-42}$. Here, the larger collection of bivalent binding antibodies expands the previous Group E and F into E1-E3 and F1-F3, which respectively target the front and backside of RBD (Fig. 1b).

By projecting each antibody's experimental measurements against each sarbecovirus onto the t-distributed stochastic neighbor embedding (t-SNE) dimension, we surprisingly found that antibodies of the same cluster have unified sarbecovirus neutralization potency and binding spectra (Fig. 1c-f, Extended Data Fig. 4a-e). Moreover, the antibodies' neutralization mechanism also tends to cluster based on the epitope distribution. Antibodies of group E1-E3 and F1 do not compete with ACE2 (Fig. $1 \mathrm{~g}$ ), which results in relatively weaker neutralizing potency compared to ACE2blocking antibody (Extended Data Fig. 5a), except that group E1 antibodies still possess high neutralization potency (Fig. 1c), suggesting a rather unique neutralization mechanism. In total, 6 clusters of antibodies were found to exhibit broad sarbecovirus neutralizing ability with diverse breadth, namely Group E1, E3, F1, F2, F3 and a subcluster of Group B (Fig 1a, 1e-f). 
Group E1 antibodies display potent neutralizing activities against human infecting sarbecoviruses, including both SARS-CoV-2 variants and SARS-CoV-1 variants (Fig. 1c, Extended Data Fig. 5b). Their epitope is fully exposed regardless of the up and down conformations of RBD and may involve a mixed protein and carbohydrate, specifically the N-linked glycan on N343, as for S309 ${ }^{19}$. Indeed this unique feature is shared among Group E antibodies, evidenced by structural analyses of BD55-3152, BD55-3546 and BD55-5840 in complex with spike proteins using cryo-electron microscopy (cryo-EM) (Fig. 2a, Extended Data Fig. 6a-b). Importantly, members of Group E1 are generally sensitive to the changes of G339, E340, T345 and especially R346, revealed by their escaping mutation profiles and structures (Fig. 2a, d). Thus, most E1 antibodies could not bind to clade 2 and 3 sarbecoviruses because of the changes of RBD antigenic sites corresponding to G339, E340 and R346, as calculated by multiple sequence alignment (MSA) (Fig. 1f, Fig. 2d). Importantly, Omicron causes considerable antibody escaping for Group E1 (Fig. 1d); however, a proportion of E1 antibodies that could tolerate G339D and N440K mutations could retain potent neutralizing ability against BA.1, BA.1.1, and BA.2, making them good therapeutics candidates (Extended Data Fig. 7).

Interestingly, despite the importance of R346 to E1 antibodies, the additional R346K carried by BA.1.1 does not readily affect their efficacy (Fig. 1d). This is foreseeable, as Arg and Lys possess similar chemical properties. In fact, SARS-CoV-1 features a Lys at the corresponding site (K333) (Fig. 2d). The structure of BD55-3152 in complex with the Omicron spike reveals that a CDRL2 Asp (D50) interacts with R346 (Fig. 2a), whereas the structure of BD55-3152 complexed with the SARS-CoV-1 spike shows that the same Asp also coordinates K333 (Extended Data Fig. 8). Nevertheless, most E1 antibodies lost binding and neutralization ability towards clade $1 \mathrm{a} / 1 \mathrm{~b}$ sarbecoviruses circulating among animals, including the bat coronavirus RaTG13 and the pangolin coronavirus Pangolin-GD (Fig. 1e, Extended Data Fig. 5f-g). This is largely because of the Thr substitution in these sarbecoviruses (Fig. 2d). In contrast to R346K, R346S/T would greatly compromise the binding activities of E1 antibodies. 
Group E2 antibodies are directed to the front chest of RBD. Previously, we solved cryoEM structures of several neutralizing antibodies in this group, including BD-744 ${ }^{43}$ (Fig. $2 b)$. These neutralizing antibodies target a relatively flat region partially exposed in the down RBDs. The E2 antibodies are sensitive to mutations of R346, A348, A352, K356, R357, L452, and I468, and these sites are largely not conserved in clade $1 \mathrm{a} / 2 / 3$ sarbecoviruses (Fig. 2d). Therefore, the E2 Antibodies usually do not have broad sarbecovirus neutralizing ability (Fig. 1e-f, Extended Data Fig. 4a-e). The L452R mutation in the Delta variant also substantially abrogates their activities (Fig. 2d). Nonetheless, most E2 antibodies can still neutralize Omicron BA.1, since Omicron BA.1 do not have mutations at the sites mentioned above (Fig. 1d, Extended Data Fig. 5c-e); however, the R346K carried by BA.1.1 would escape E2 antibodies as well (Fig 2d), such as Brii-198.

Group E3 antibodies, such as S2H9720, bind to the left flank of RBD (Fig. 2c). As this epitope region is deeply buried within the spike trimer, the E3 antibodies can only bind when the RBD adopts a not only up but also wide open conformation. Likely because of this, the neutralizing activities of group E3 antibodies are relatively modest. Critically sites recognized by E3 antibodies include R357, T393, Y396, D428, K462, S514, E516 and L518, all well conserved in most sarbecoviruses (Fig. 2d); thus, the E3 antibodies display great neutralization breadth (Fig. 1f, Extended Data Fig. 4a-e). Interestingly, though not escaped by Omicron variants, their overall neutralizing activities are reduced (Fig. 1d). The neutralization mechanisms of S2H97 and other antibodies targeting the cryptic sites in RBD have been partly attributed to their ability to disrupt the spike trimer's prefusion state, as these antibodies demand the extensive opening of the RBD to engage their respective binding sites ${ }^{20,44-46}$. Their reduced activities towards Omicron could thus be caused by the more stabilized prefusion structure of Omicron spike ${ }^{47}$. 
and can only bind to the up RBDs (Fig. 1b). Members of the F1 group, such as $\mathrm{CR} 3022^{21}$ and $\mathrm{S} 304^{22}$, also require a wide open RBD to engage and do not directly block ACE2, therefore displaying weak neutralizing activities in general (Fig. 1c). Escape hotspots for this group include SARS-CoV-2 residues 383-386, 390, and 391 (Fig. 3a, d). Most of these sites are conserved in all sarbecoviruses, except an alanine on 384 in clade 1a, which is also tolerated (Fig. 3d). Due to their intrinsic weak neutralizing activities and the triple mutations on S371, S373, S375 of Omicron, we found that the F1 antibodies virtually have no neutralizing power against Omicron variants (Fig. 1d, Extended Data Fig. 5c-e).

The epitopes for group F2 antibodies are shifted upward compared with F1 (Fig. 3b). We solved the cryo-EM structure of two representative antibodies in this group, BD551239 and BD55-3500, both in complexes with the Omicron spike (Fig. 3b, Extended Data Fig. 6c). Structural analyses showed that although these antibodies do not directly target the ACE2-binding residues, both project towards where ACE2 would be lodged and therefore exclude ACE2 through steric hindrance, like DH104723 (Fig. 3b). Group F2 antibodies can be escaped by RBD mutation involving T376, K378, and R408 (Fig. 3d). Indeed, these residues are all at the heart of BD55-1239's and BD55-3500's epitopes. These sites are fairly conserved across sarbecoviruses (Fig. 3b, d), and the neutralization breadth of group F2 antibodies is comparable to that of E3 and F1 (Fig. 1e-f). A notable exception is the corresponding K378Q substitution in BM48-31, which might compromise the binding of many F2 antibodies (Extended Data Fig. 4d). Despite the extraordinary breadth and potency against sarbecovirus, Group F2 antibodies suffer great neutralization efficacy reduction against Omicron, mostly due to the triple mutations on S371, S373, S375 (Fig 1d). Importantly, mutation T376A and R408S harbored by Omicron BA.2 completely abolished the neutralizing capacity of F2 antibodies (Fig 1d, Extended Data Fig. 5e).

Group F3 Antibodies reach further towards the ACE2-binding site, such as S2X25926 and ADG-2 ${ }^{24}$ (Fig. 1b). Cryo-EM structures of BD55-4637 complexed with the 
Omicron spike and BD55-3372 with the Delta spike reveal that F3 antibodies interact with several ACE2-binding residues (Fig. 3c, Extended Data Fig. 6d), and therefore directly compete with ACE2. Major escape sites for this group of antibodies include D405, R408, V503, G504, and Y508 (Fig. 3d). Given the fact that D405, G504, and Y508 are not conserved in clade 2 sarbecoviruses, F3 antibodies cannot bind to nonACE2 utilizing clade 2 sarbecovirus (Extended Data Fig. 4e), but showed good neutralization breadth against clade $1 \mathrm{a} / \mathrm{b} / 2$ sarbecovirus (Fig. 1e-f). Similar to Group E1, a proportion of F3 antibodies showed potent neutralization against Omicron BA.1, despite the Y505H mutation carried by Omicron (Fig. 1d, 3d). Moreover, several elite members of BA.1 tolerating F3 antibodies could also overcome the D405N and R408S mutations of BA.2, making them good therapeutic drug candidates (Fig 1d, Extended Data Fig. 7).

Notably, a unique subcluster of Group B antibodies also showed broad-spectrum sarbecovirus neutralizing capability (Fig 1c). Most Group B antibodies are SARS-CoV2 specific since their major escaping mutations consist of E484 and F486, which are not conserved in sarbecovirus clade (Extended Data Fig. 9); however, the rare subcluster B', featured by S2K146 25 , displayed skewed escaping mutation profiles toward N487 and Y489, which are highly conserved in clade 1a/1b/3 (Extended Data Fig. 9), making members of B' exhibits similar breadth as F3 antibodies. Sadly, most B' antibodies failed to neutralize Omicron, except for S2K146 ${ }^{6}$ (Fig. 1d).

The above analyses indicate that each epitope group's mutational escape hotspots are closely related to the antibodies' sarbecovirus reactivity breadth. To further extrapolate this observation, we simulated each antibody's sarbecovirus RBD binding spectrum based on its escaping mutation profile and the corresponding MSA results of sarbecovirus RBDs (Extended Data Fig. 10a-e). Surprisingly, the simulated spectrum well matches that obtained from ELISA (Extended Data Fig. 4a-e), suggesting that the antibody's breadth is mostly governed by the degree of conservation of its major mutational escaping sites, and the escaping mutation profile of an antibody could be 
used to predict its sarbecovirus binding spectrum.

Importantly, even broad sarbecovirus neutralizing antibodies are largely escaped by Omicron (Fig. 1b), which is consistent with that Omicron could greatly reduce the neutralization efficacy of vaccinated SARS convalescents' plasma (Extended Data Fig. 1c). This supports the speculation that Omicron does not originate from zoonotic spillover but immune selection pressure. Indeed, multiple top antibody-escaping mutations inferred from yeast display have appeared on Omicron variants, including K417N, E484A, Q490R, G446S, G339D, R346K, N440K, T376A, D405N and R408S (Supplementary Data 3). Especially BA.2, which has evolved multiple mutations on the sarbecovirus conserved amino acids (D405N, R408S and T376A) that could cause pinpoint escapes of most F2 and partial F3 broad-spectrum neutralizing antibodies (Fig. 1d, Extended Data Fig. 3). Nevertheless, BA.2 lacks the G446S mutation, and thus part of group D antibodies that target the linear 440-449 loop retained their neutralization capability against BA.2, such as REGEN-1098748 and AZD106149 (Fig. 1d).

We have identified a large panel of potent sarbecovirus neutralizing antibodies that stay effective to Omicron variants, mainly belonging to Group E1, F2 and F3. To further examine their potential as antibody therapeutics, we analyzed these antibodies' authentic virus neutralization potency against Omicron (BA.1) as well as their binding affinity against sarbecovirus RBDs, along with well-recognized broad-spectrum neutralizing antibody serving as controls (Fig. 4a, Supplementary Data 4). Several antibodies, such as BD55-5840 (E1), BD55-3546 (E1), BD55-5640 (F2), BD55-5514 (F3) and BD55-5483 (F3), stand out as promising drug candidates (Fig. 4a).

In addition, pairing non-competing antibodies into cocktails is a promising strategy to reduce the chance of mutation-induced antibody evasion. As the epitopes of the E1 antibodies are located on the opposite sides of RBD compared to F2 and F3, we envision that many E1 antibodies can function in combinations with an F2 or F3 counterpart. Indeed, several plausible strategies can be designed based on the structural 
information presented in this study. For example, overlaying the targeting RBDs in the BD55-5840 (E1) and BD55-3500 (F2) cryo-EM structures demonstrate that they can readily bind to the Omicron RBD simultaneously (Fig. 4b), whereas BD55-3546 (E1) can bind concurrently with BD55-3372 (F3) on the Delta RBD (Fig. 4c). From a mechanistic point of view, E1 antibodies do not directly impede ACE2 but can engage both the up and down RBDs, whereas F2/F3 antibodies only bind to the up RBDs but promptly blocks ACE2. Due to these complementary properties, they might synergize in an ideal way to achieve maximized breadth and potency.

Our results could also guide the development of broad sarbecovirus vaccines. According to our analyses, certain neutralizing antibodies, such as those belonging to E1, F2, F3 epitope groups, should be the ideal inducing targets of broad sarbecovirus vaccines, since their neutralization potency and breadth far exceeds the antibodies in other epitope groups. Also, the overall mean escaping mutation profile for each epitope group has great implications for rational vaccine antigen design (Supplementary Data 3). It could offer the exact mutation combination that should be used to avoid stimulating a certain antibody group, such as a vaccine booster dose using a spike protein with $\mathrm{K} 417 \mathrm{D} / \mathrm{F} 456 \mathrm{~K} / \mathrm{A} 475 \mathrm{R}$ mutations should completely block the stimulation of Group A antibodies. Together, our results provide a comprehensive understanding of the RBD epitopes that can induce broad sarbecovirus neutralizing antibodies, which offers critical instructions for sarbecovirus antibody therapeutics and the antigen design of broad-spectrum sarbecovirus vaccines.

\section{Reference}

1 Viana, R. et al. Rapid epidemic expansion of the SARS-CoV-2 Omicron variant in southern Africa. Nature, doi:10.1038/s41586-022-04411-y (2022).

2 Lu, L. et al. Neutralization of SARS-CoV-2 Omicron variant by sera from BNT162b2 or Coronavac vaccine recipients. Clin Infect Dis, doi:10.1093/cid/ciab1041 (2021).

3 Cele, S. et al. Omicron extensively but incompletely escapes Pfizer BNT162b2 neutralization. Nature, doi:10.1038/s41586-021-04387-1 (2021).

4 Collie, S., Champion, J., Moultrie, H., Bekker, L. G. \& Gray, G. Effectiveness of BNT162b2 Vaccine 
against Omicron Variant in South Africa. $N$ Engl J Med 386, 494-496, doi:10.1056/NEJMc2119270 (2022).

5 Dejnirattisai, W. et al. SARS-CoV-2 Omicron-B.1.1.529 leads to widespread escape from neutralizing antibody responses. Cell, doi:10.1016/j.cell.2021.12.046 (2022).

6 Cameroni, E. et al. Broadly neutralizing antibodies overcome SARS-CoV-2 Omicron antigenic shift. Nature, doi:10.1038/s41586-021-04386-2 (2021).

7 Cao, Y. et al. Omicron escapes the majority of existing SARS-CoV-2 neutralizing antibodies. Nature, doi:10.1038/s41586-021-04385-3 (2021).

8 Liu, L. et al. Striking Antibody Evasion Manifested by the Omicron Variant of SARS-CoV-2. Nature, doi:10.1038/s41586-021-04388-0 (2021).

9 Planas, D. et al. Considerable escape of SARS-CoV-2 Omicron to antibody neutralization. Nature, doi:10.1038/s41586-021-04389-z (2021). Lyngse, F. P. et al. Transmission of SARS-CoV-2 Omicron VOC subvariants BA.1 and BA.2: Evidence from Danish Households. medRxiv (2022).

11 Corti, D., Purcell, L. A., Snell, G. \& Veesler, D. Tackling COVID-19 with neutralizing monoclonal antibodies. Cell 184, 3086-3108, doi:10.1016/j.cell.2021.05.005 (2021).

12 Cohen, A. A. et al. Mosaic nanoparticles elicit cross-reactive immune responses to zoonotic coronaviruses in mice. Science 371, 735-741, doi:10.1126/science.abf6840 (2021).

13 Martinez, D. R. et al. Chimeric spike mRNA vaccines protect against Sarbecovirus challenge in mice. Science 373, 991-998, doi:10.1126/science.abi4506 (2021).

14 Walls, A. C. et al. Elicitation of broadly protective sarbecovirus immunity by receptor-binding domain nanoparticle vaccines. Cell 184, 5432-5447 e5416, doi:10.1016/j.cell.2021.09.015 (2021).

15 Starr, T. N. et al. Deep Mutational Scanning of SARS-CoV-2 Receptor Binding Domain Reveals Constraints on Folding and ACE2 Binding. Cell 182, 1295-1310 e1220, doi:10.1016/j.cell.2020.08.012 (2020).

16 Greaney, A. J. et al. Comprehensive mapping of mutations in the SARS-CoV-2 receptor-binding domain that affect recognition by polyclonal human plasma antibodies. Cell Host Microbe 29, 463-476 e466, doi:10.1016/j.chom.2021.02.003 (2021).

17 Greaney, A. J. et al. Complete Mapping of Mutations to the SARS-CoV-2 Spike Receptor-Binding Domain that Escape Antibody Recognition. Cell Host Microbe 29, 44-57 e49, doi:10.1016/j.chom.2020.11.007 (2021).

18 Starr, T. N., Greaney, A. J., Dingens, A. S. \& Bloom, J. D. Complete map of SARS-CoV-2 RBD mutations that escape the monoclonal antibody LY-CoV555 and its cocktail with LY-CoV016. Cell Rep Med 2, 100255, doi:10.1016/j.xcrm.2021.100255 (2021).

19 Pinto, D. et al. Cross-neutralization of SARS-CoV-2 by a human monoclonal SARS-CoV antibody. Nature 583, 290-295, doi:10.1038/s41586-020-2349-y (2020).

20 Starr, T. N. et al. SARS-CoV-2 RBD antibodies that maximize breadth and resistance to escape. Nature 597, 97-102, doi:10.1038/s41586-021-03807-6 (2021).

21 Yuan, M. et al. A highly conserved cryptic epitope in the receptor binding domains of SARSCoV-2 and SARS-CoV. Science 368, 630-633, doi:10.1126/science.abb7269 (2020). Receptor-Binding Domain by Structure-Guided High-Resolution Serology. Cell 183, 1024-1042 e1021, doi:10.1016/j.cell.2020.09.037 (2020). 
Martinez, D. R. et al. A broadly cross-reactive antibody neutralizes and protects against sarbecovirus challenge in mice. Sci Transl Med 14, eabj7125, doi:10.1126/scitranslmed.abj7125 (2022). Rappazzo, C. G. et al. Broad and potent activity against SARS-like viruses by an engineered human monoclonal antibody. Science 371, 823-829, doi:10.1126/science.abf4830 (2021). Park, Y. J. et al. Antibody-mediated broad sarbecovirus neutralization through ACE2 molecular mimicry. Science 375, 449-454, doi:10.1126/science.abm8143 (2022). Tortorici, M. A. et al. Broad sarbecovirus neutralization by a human monoclonal antibody. Nature 597, 103-108, doi:10.1038/s41586-021-03817-4 (2021).

LV, H. et al. Cross-reactive Antibody Response between SARS-CoV-2 and SARS-CoV Infections. Cell Rep 31, 107725, doi:10.1016/j.celrep.2020.107725 (2020). Jette, C. A. et al. Broad cross-reactivity across sarbecoviruses exhibited by a subset of COVID19 donor-derived neutralizing antibodies. Cell Rep 36, 109760, doi:10.1016/j.celrep.2021.109760 (2021).

Liu, H. et al. A combination of cross-neutralizing antibodies synergizes to prevent SARS-CoV-2 and SARS-CoV pseudovirus infection. Cell Host Microbe 29, 806-818 e806, doi:10.1016/j.chom.2021.04.005 (2021).

Wec, A. Z. et al. Broad neutralization of SARS-related viruses by human monoclonal antibodies. Science 369, 731-736, doi:10.1126/science.abc7424 (2020).

Burnett, D. L. et al. Immunizations with diverse sarbecovirus receptor-binding domains elicit SARS-CoV-2 neutralizing antibodies against a conserved site of vulnerability. Immunity 54, 2908-2921 e2906, doi:10.1016/j.immuni.2021.10.019 (2021).

Wang, P. et al. A monoclonal antibody that neutralizes SARS-CoV-2 variants, SARS-CoV, and other sarbecoviruses. Emerg Microbes Infect 11, 147-157, doi:10.1080/22221751.2021.2011623 (2022). elite neutralizers. Cell Host Microbe 30, 69-82 e10, doi:10.1016/j.chom.2021.12.010 (2022).

Onodera, T. et al. A SARS-CoV-2 antibody broadly neutralizes SARS-related coronaviruses and variants by coordinated recognition of a virus-vulnerable site. Immunity 54, 2385-2398 e2310, doi:10.1016/j.immuni.2021.08.025 (2021).

Tan, C. W. et al. Pan-Sarbecovirus Neutralizing Antibodies in BNT162b2-Immunized SARS-CoV1 Survivors. N Engl J Med 385, 1401-1406, doi:10.1056/NEJMoa2108453 (2021).

Cao, Y. et al. Potent Neutralizing Antibodies against SARS-CoV-2 Identified by High-Throughput Single-Cell Sequencing of Convalescent Patients' B Cells. Cell 182, 73-84 e16, doi:10.1016/j.cell.2020.05.025 (2020).

Scheid, J. F. et al. B cell genomics behind cross-neutralization of SARS-CoV-2 variants and SARSCoV. Cell 184, 3205-3221 e3224, doi:10.1016/j.cell.2021.04.032 (2021).

Cao, Y. et al. Humoral immune response to circulating SARS-CoV-2 variants elicited by inactivated and RBD-subunit vaccines. Cell Res 31, 732-741, doi:10.1038/s41422-021-00514-9 (2021).

Greaney, A. J. et al. Mapping mutations to the SARS-CoV-2 RBD that escape binding by different classes of antibodies. Nat Commun 12, 4196, doi:10.1038/s41467-021-24435-8 (2021).

Dejnirattisai, W. et al. The antigenic anatomy of SARS-CoV-2 receptor binding domain. Cell 184, 2183-2200 e2122, doi:10.1016/j.cell.2021.02.032 (2021). 
41 Barnes, C. O. et al. SARS-CoV-2 neutralizing antibody structures inform therapeutic strategies. Nature 588, 682-687, doi:10.1038/s41586-020-2852-1 (2020).

42 Yuan, M. et al. Structural and functional ramifications of antigenic drift in recent SARS-CoV-2 variants. Science 373, 818-823, doi:10.1126/science.abh1139 (2021).

43 Du, S. et al. Structures of SARS-CoV-2 B.1.351 neutralizing antibodies provide insights into cocktail design against concerning variants. Cell Res 31, 1130-1133, doi:10.1038/s41422-02100555-0 (2021).

$44 \mathrm{Li}$, T. et al. Cross-neutralizing antibodies bind a SARS-CoV-2 cryptic site and resist circulating variants. Nat Commun 12, 5652, doi:10.1038/s41467-021-25997-3 (2021).

45 Huo, J. et al. Neutralization of SARS-CoV-2 by Destruction of the Prefusion Spike. Cell Host Microbe 28, 445-454 e446, doi:10.1016/j.chom.2020.06.010 (2020).

46 Wrobel, A. G. et al. Antibody-mediated disruption of the SARS-CoV-2 spike glycoprotein. Nat Commun 11, 5337, doi:10.1038/s41467-020-19146-5 (2020).

47 Cui, Z. et al. Structural and functional characterizations of infectivity and immune evasion of SARS-CoV-2 Omicron. Cell, doi:10.1016/j.cell.2022.01.019.

48 Hansen, J. et al. Studies in humanized mice and convalescent humans yield a SARS-CoV-2 antibody cocktail. Science 369, 1010-1014, doi:10.1126/science.abd0827 (2020).

49 Dong, J. et al. Genetic and structural basis for SARS-CoV-2 variant neutralization by a twoantibody cocktail. Nat Microbio/ 6, 1233-1244, doi:10.1038/s41564-021-00972-2 (2021).

\section{Methods}

\section{Antibody isolation and recombinant production}

SARS-CoV-1 and SARS-CoV-2 RBD cross-binding memory B cells were isolated from PBMC of SARS-CoV-1 convalescents who received SARS-CoV-2 vaccine. Briefly, CD19+ B cells were isolated from PBMC with EasySep ${ }^{\mathrm{TM}}$ Human CD19 Positive Selection Kit II (STEMCELL, 17854). B cells were then stained with FITC anti-human CD19 antibody (BioLegend, 392508), FITC anti-human CD20 antibody (BioLegend, 302304), Brilliant Violet 421 $1^{\mathrm{TM}}$ anti-human CD27 antibody (BioLegend, 302824), PE/Cyanine7 anti-human IgM antibody (BioLegend, 314532), biotinylated Ovalbumin (SinoBiological) conjugated with Brilliant Violet 605 405229), SARS-CoV-1 biotinylated RBD protein (His \& AVI Tag) (SinoBiological, 40634-V27H-B) conjugated with PE-streptavidin (BioLegend, 405204), SARS-CoV-2 biotinylated RBD protein (His \& AVI Tag) (SinoBiological, 40592-V27H-B) 
conjugated with APC-streptavidin (BioLegend, 405207), and 7-AAD (Invitrogen, 006993-50). CD19/CD20+, CD27+, IgM-, OVA-, SARS-COV-1 RBD+, and SARS-CoV$2 \mathrm{RBD}+$ were sorted with MoFlo Astrios EQ Cell Sorter (Beckman Coulter). FACS data were analyzed using FlowJo $\mathrm{J}^{\mathrm{TM}}$ v10.8 (BD Biosciences).

Sorted SARS-CoV-1 and SARS-CoV-2 RBD cross-binding B cells were then processed with Chromium Next GEM Single Cell V(D)J Reagent Kits v1.1 following the manufacturer's user guide (10x Genomics, CG000208). Briefly, Cells sorted were resuspend in PBS after centrifugation. Gel beads-in-emulsion (GEMs) were obtained with 10X Chromium controller and then subjected to reverse transcription (RT). After GEM-RT clean up, RT products were subject to preamplification. After amplification and purification with SPRIselect Reagent Kit (Beckman Coulter, B23318) of RT products, B cell receptor (BCR) sequence (paired V(D)J) were enriched with 10X BCR primers. After library preparation, libraries were sequenced by Novaseq 6000 platform running Novaseq 6000 S4 Reagent Kit v1.5 300 cycles (Illumina, 20028312) or NovaSeq XP 4-Lane Kit v1.5 (Illumina, 20043131).

After sequencing and data processing, monoclonal antibodies were expressed as recombinant human IgG1. Briefly, HEK293F cells (Thermo Fisher, R79007) were transiently transfected with heavy and light chain expression vectors. The secreted monoclonal antibodies from cultured cells were purified by protein A affinity chromatography. The specificities of these antibodies were determined by ELISA.

\section{Antibody sequence analysis}

The antibody sequences obtained from 10X Genomics V(D)J sequencing were aligned to GRCh38 reference and assembled as immunoglobulin contigs by the Cell Ranger (v6.1.1) pipeline. Non-productive contigs and B cells that had multiple heavy chain or light chain contigs were filtered out of the analysis. V(D)J gene annotation was performed using NCBI IgBlast (v1.17.1) with the IMGT reference. Mutations on V(D)J nucleotide sequences were calculated by using the igpipeline, which compared the 
sequences to the closest germline genes and counted the number of different nucleotides. For antibodies from public sources whose original sequencing nucleotide sequences were not all accessible, the antibody amino acid sequences were annotated by IMGT/DomainGapAlign ${ }^{50}$ (v4.10.2) with default parameters. The V-J pairs were visualized by R package circlize (v0.4.10).

\section{High-throughput antibody-escape mutation profiling}

The previously described high-throughput MACS (magnetic-activated cell sorting)based antibody-escape mutation profiling system was used to characterize RBD escaping mutation profile for neutralizing antibodies. Briefly, duplicate RBD mutant libraries were constructed based on Wuhan-Hu-1 RBD sequence (GenBank: MN908947, residues N331-T531), theoretically containing 3819 possible amino acid mutations. Each RBD mutant was barcoded with a unique 26-neuclotide (N26) and only ACE2 binding variants were enriched for downstream experiment.

For antibody escape profiling, yeast libraries were induced overnight for RBD expression and washed followed by two rounds of Protein A antibody based negative selection and MYC-tag based positive selection to enrich RBD expressing cells. Protein A antibody conjugated products were prepared following the protocol of Dynabeads Protein A (Thermo Fisher, 10008D) and incubated with induced yeast libraries at room temperature. MYC-tag based positive selection was performed according to the manufacturer's protocol (Thermo Fisher, 88843).

After three rounds of sequential cell sorting, the obtained yeast cells were recovered overnight. Plasmids were extracted from pre- and post-sort yeast populations by 96Well Plate Yeast Plasmid Preps Kit (Coolaber, PE053). The extracted plasmids were then used to amplify N26 barcode sequences by PCR. The final PCR products were purified with AMPure XP magnetic beads (Beckman Coulter, A63882) and 75bp singleend sequencing was performed on an Illumina Nextseq 500 platform. 


\section{Processing of deep mutational scanning data}

Single-end Illumina sequencing reads were processed as previously described. Briefly, reads were trimmed into 16 or 26 bp and aligned to the reference barcode-variant dictionary with dms_variants package (v0.8.9). Escape scores of variants were calculated as $\mathrm{F} \times\left(\mathrm{n}_{\mathrm{X}, \mathrm{ab}} / \mathrm{N}_{\mathrm{ab}}\right) /\left(\mathrm{n}_{\mathrm{X}, \mathrm{ref}} / \mathrm{N}_{\text {ref }}\right)$, where $\mathrm{n}_{\mathrm{X}, \mathrm{ab}}$ and $\mathrm{n}_{\mathrm{X}}$,ref is the number of reads representing variant $\mathrm{X}$, and $\mathrm{N}_{\mathrm{ab}}$ and $\mathrm{N}_{\mathrm{ref}}$ are the total number of valid reads in antibodyselected (ab) and reference (ref) library, respectively. F is a scale factor defined as the 99th percentiles of escape fraction ratios. Variants detected by less than 6 reads in the reference library were removed to avoid sampling noise. Variants containing mutations with ACE2 binding below -2.35 or RBD expression below -1 were removed as well, according to data previously reported. Finally, global epistasis models were built using dms_variants package to estimate mutation escape scores. For most antibodies, at least two independent assays are conducted and single mutation escape scores are averaged across all experiments that pass quality control.

\section{Antibody clustering and visualization}

Site total escape scores, defined as the sum of escape scores of all mutations at a particular site on RBD, were used to evaluate the impact of mutations on each site for each antibody. Each of these scores is considered as a feature of a certain antibody and used to construct a feature matrix $\mathbf{A}_{\mathrm{N} \times \mathrm{M}}$ for downstream analysis, where $\mathrm{N}$ is the number of antibodies and $\mathrm{M}$ is the number of features (valid sites). Informative sites were selected using sklearn.feature_selection.VarianceThreshold of scikit-learn Python package (v0.24.2) with the variance threshold as 0.1 . Then, the selected features were L2-normalized across antibodies using sklearn.preprocessing.normalize. The resulting matrix is referred as $\mathbf{A}^{\prime} \mathbf{N} \times \mathbf{M}^{\prime}$, where $\mathrm{M}^{\prime}$ is the number of selected features. The dissimilarity of two antibodies $\mathrm{i}, \mathrm{j}$ is defined as $1-\operatorname{Corr}\left(\mathbf{A}_{\mathrm{i}}, \mathbf{A}_{\mathrm{j}}\right)$, where $\operatorname{Corr}(\mathbf{x}, \mathbf{y})$ is the Pearson's correlation coefficient of vector $\mathbf{x}$ and $\mathbf{y}$, i.e. $\operatorname{Corr}(\mathbf{x}, \mathbf{y})=\mathbf{x} \cdot \mathbf{y} /|\mathbf{x}||\mathbf{y}|$. We used sklearn.manifold.MDS to reduce the number of features from $\mathrm{M}^{\prime}$ to $\mathrm{D}=20$ with multidimensional scaling under the above metric. Antibodies are clustered into 10 epitope groups using sklearn.cluster.KMeans of scikit-learn in the resulting D- 
504

505

506

507

508

509

510

511

512

513

514

515

516

517

518

519

520

521

522

523

524

525

526

527

528

529

530

531

dimensional feature space. Finally, these D-dimensional representations of antibodies were further embedded into two-dimensional space for visualization with t-SNE using sklearn.manifold.TSNE of scikit-learn. All t-SNE plots were generated by R package ggplot2 (v3.3.3).

\section{Pseudovirus neutralization assay}

SARS-CoV-2 spike (GenBank: MN908947), Pangolin-GD spike (GISAID: EPI_ISL_410721), RaTG13 spike (GISAID: EPI_ISL_402131), SARS-COV-1 spike (GenBank: AY278491), Omicron BA.1 spike (A67V, H69del, V70del, T95I, G142D, V143del, Y144del, Y145del, N211del, L212I, ins214EPE, G339D, S371L, S373P, S375F, K417N, N440K, G446S, S477N, T478K, E484A, Q493R, G496S, Q498R, N501Y, Y505H, T547K, D614G, H655Y, N679K, P681H, N764K, D796Y, N856K, Q954H, N969K, L981F), BA.2 spike (GISAID: EPI_ISL_7580387), BA.1.1 spike (BA.1+R346K), plasmid is constructed into pcDNA3.1 vector. $\mathrm{G}^{*} \Delta \mathrm{G}-\mathrm{VSV}$ virus (VSV G pseudotyped virus, Kerafast) is used to infect 293T cells (American Type Culture Collection [ATCC], CRL-3216), and spike protein expressing plasmid was used for transfection at the same time. After culture, the supernatant containing pseudovirus was harvested, filtered, aliquoted, and frozen at $-80^{\circ} \mathrm{C}$ for further use.

Pseudovirus detection of PCoV-GD and RaTG13 was performed in 293T cells overexpressing human angiotensin-converting enzyme 2 (293T-hACE2 cells). Other pseudovirus neutralization assays were performed using the Huh-7 cell line (Japanese Collection of Research Bioresources [JCRB], 0403).

Monoclonal antibodies or plasma were serially diluted (5-fold or 3-fold) in DMEM (Hyclone, SH30243.01) and mixed with pseudovirus in 96-well plates. After incubation at $5 \% \mathrm{CO}_{2}$ and $37^{\circ} \mathrm{C}$ for $1 \mathrm{~h}$, digested Huh-7 cell (Japanese Collection of Research Bioresources [JCRB], 0403) or 293T-hACE2 cells (AmericanTypeCultureCollection[ATCC],CRL-3216) were seeded. After 24 hours of culture, supernatant was discarded and D-luciferin reagent (PerkinElmer, 6066769) was 
added to react in the dark, and the luminescence value was detected using a microplate spectrophotometer (PerkinElmer, HH3400). IC50 was determined by a four-parameter logistic regression model.

\section{Authentic virus neutralization}

SARS-CoV-2 and SARS-CoV-2 variants neutralization by plasma were performed using cytopathic effect (CPE) assay. Plasma samples were inactivated at $56^{\circ} \mathrm{C}$ for $0.5 \mathrm{~h}$. Plasma samples were serially diluted ( 2 fold) in the 96 -well plates with cell culture medium and mixed with $100 \mathrm{TCID} 50$ virus, and then incubated at $37^{\circ} \mathrm{C}, 5 \% \mathrm{CO}_{2}$ for 2 hours. About $10^{4}$ digested Vero cells (ATCC, CCL-81) were seeded in plates and cultured for 5 days. CPE of each well was recorded under microscopes. Assays for each sample were replicated and the neutralization titer was calculated by the SpearmanKarber method. All experiments were performed in a Biosafety Level 3 laboratory.

\section{ELISA}

To detect the broad-spectrum binding of the antibodies among Sarbecovirus, we entrusted SinoBiological Technology Co., Ltd. to synthesize a panel of 20 sarbecovirus RBDs (Supplementary Table 3). According to the sequence of 20 RBDs, a set of nested primers was designed. The coding sequences were obtained by the overlap-PCR with a 6xHis tag sequence to facilitate protein purification. The purified PCR products were ligated to the secretory expression vector pCMV3 with CMV promoter, and then transformed into E. coli competent cells XL1-blue. Monoclones with correct transformation were cultured and expanded, and plasmids were extracted. Healthy HEK293 cells were passaged into a new cell culture and grown in suspension at $37^{\circ} \mathrm{C}$, $120 \mathrm{RPM}, 8 \% \mathrm{CO} 2$ to logarithmic growth phase and transfected with the recombinant constructs by using liposomal vesicles as DNA carrier. After transfection, the cell cultures were followed to assess the kinetics of cell growth and viability for 7 days. The cell expression supernatant was collected, and after centrifugation, passed through a Ni column for affinity purification. The molecular size and purity of eluted protein was confirmed by SDS-PAGE. Production lot numbers and concentration information 
of the 20 Sarbecovirus proteins are shown in Suppplemenatary Table 4. The WT RBD in the article is SARS-CoV-2 (2019-nCoV) Spike RBD-His Recombinant Protein (SinoBiological, 40592-V08H).

A panel of 21 sarbecovirus RBDs (supplementary table3) in PBS was pre-coated onto ELISA Plates (NEST, 514201) at $4{ }^{\circ} \mathrm{C}$ overnight. The plates were washed and blocked. Then $1 \mu \mathrm{g} / \mathrm{ml}$ purified antibodies or serially diluted antibodies were added and incubated at room temperature (RT) for 20min. Next, Peroxidase-conjugated AffiniPure Goat Anti-Human IgG (H+L) (JACKSON, 109-035-003) was applied and incubated at RT for $15 \mathrm{~min}$. Tetramethylbenzidine (TMB) (Solarbio, 54827-17-7) was added onto the plates. The reaction was terminated by $2 \mathrm{M} \mathrm{H}_{2} \mathrm{SO}_{4}$ after $10 \mathrm{~min}$ incubation. Absorbance was measured at $450 \mathrm{~nm}$ using Ensight Multimode Plate Reader (PerkinElmer, HH3400). ELISA OD450 measurements at different antibody concentration for a particular antibody-antigen pair are fit to the model $y=A c^{n} /\left(c^{n}+E^{n}\right)$ using $R$ package mosaic (v1.8.3), where $\mathrm{y}$ is OD450 values and $\mathrm{c}$ is corresponding antibody concentration. $\mathrm{A}, \mathrm{E}, \mathrm{n}$ are parameters, where $\mathrm{E}$ is the desired $\mathrm{EC}_{50}$ value for the specific antibody and antigen.

\section{Antibody-ACE2 competition for RBD}

mFC-WT-RBD (Sino Biological, 40592-V05H) protein in PBS was immobilized on the ELISA plates at $4{ }^{\circ} \mathrm{C}$ overnight. The coating solution was removed and washed three times by PBST and the plates were then blocked for $2 \mathrm{~h}$. After blocking, the plates were washed five times, and the mixture of ACE2-his (Sino Biological, 10108$105 \mathrm{H}$ ) and serially diluted competitor antibodies was added followed by $30 \mathrm{~min}$ incubation at RT. Then anti-his-HRP (Proteintech, HRP-66005) was added into each well for another 20min incubation at RT. After washing the plates for five times, Tetramethylbenzidine (TMB) (Solarbio, 54827-17-7) was added into each well. After $10 \mathrm{~min}$, the reaction was terminated by $2 \mathrm{M} \mathrm{H}_{2} \mathrm{SO}_{4}$. Absorbance was measured at 450 nm using Ensight Multimode Plate Reader ( PerkinElmer, HH3400). The ACE2 competition coefficient is calculated as $(\mathrm{B}-\mathrm{A}) / \mathrm{B}$, where $\mathrm{B}$ is the OD450 value under 
$0.15 \mathrm{ug} / \mathrm{ml}$ antibody concentration and $\mathrm{A}$ is the $\mathrm{OD} 450$ value under $3 \mathrm{ug} / \mathrm{ml}$ antibody concentration.

\section{Biolayer Interferometry}

Biolayer interferometry assays were performed on Octet $\AA$ RED96 Protein Analysis System (Fortebio) according to the manufacturer's instruction. The kinetics assays were conducted with protein A biosensor (ForteBio 18-5010). (1) Sensor check: sensors immersed $10 \mathrm{~min}$ in buffer alone (buffer ForteBio 18-1105). (2) Baseline: sensors immersed $30 \mathrm{~s}$ in buffer alone. (3) Loading: sensors immersed $300 \mathrm{~s}$ with antibody at $2 \mu \mathrm{g} / \mathrm{ml}$ to capture $\mathrm{Ab}$, with a threshold of $0.4 \mathrm{~nm}$. (4) Baseline 2: sensors immersed $120 \mathrm{~s}$ in buffer alone. (5) Antigen association: sensors immersed $60 \mathrm{~s}$ with serial dilutions of RBD or its variants at 75-5 nM. (6) Antigen dissociation: sensors immersed $600 \mathrm{~s}$ in buffer alone. (7) Sensor regeneration: sensors immersed $30 \mathrm{~s}$ in regeneration buffer (10 mM Glycine- $\mathrm{HCl}, \mathrm{pH} 1.5)$, then immersed $30 \mathrm{~s}$ in buffer. Repeat for 2 more times. Data were collected with Octet Acquisition 9.0 (Fortebio) and analyzed by Octet Analysis 9.0 (Fortebio) and Octet Analysis Studio 12.2 (Fortebio).

\section{Protein expression and purification for cryo-EM study}

The S6P expression construct encoding the SARS-CoV-2 spike ectodomain (residues 1-1208) with six stabilizing Pro substitutions (F817P, A892P, A899P, A942P, K986P, and V987P) and a "GSAS" substitution for the furin cleavage site (residues 682-685) was previously described ${ }^{51}$. The Delta specific mutations (T19R, G142D, 156del, 157del, R158G, L452R, T478K, D614G, P681R, D950N) were introduced into this construct using site-directed mutagenesis. The S6P expression construct containing the Omicron mutations (A67V, H69del, V70del, T95I, G142D, V143del, Y144del, Y145del, N211del, L212I, ins214EPE, G339D, S371L, S373P, S375F, K417N, N440K, G446S, S477N, T478K, E484A, Q493R, G496S, Q498R, N501Y, Y505H, T547K, D614G, 
H655Y, N679K, P681H, N764K, D796Y, N856K, Q954H, N969K, L981F) were assembled from three synthesized DNA fragments. The expression construct encoding the SARS-CoV spike ectodomain (residues $1-1195)^{52}$ was kindly provided by Prof. X. Wang (Tsinghua university), and two stabilizing Pro substitutions (K968P, V969P) was engineered into this construct using mutagenesis. For protein production, these expression plasmids, as well as the plasmids encoding the antigen-binding fragments (Fabs) of the antibodies described in this paper, were transfected into the HEK293F cells using polyethylenimine (Polysciences). The conditioned media were harvested and concentrated using a Hydrosart ultrafilter (Sartorius), and exchanged into the binding buffer $(25 \mathrm{mM}$ Tris, $\mathrm{pH} 8.0$, and $200 \mathrm{mM} \mathrm{NaCl}$ ). Protein purifications were performed using the Ni-NTA affinity method, followed by gel filtration chromatographies using either a Superose 6 increase column (for the spike proteins) or a Superose 200 increase column (for the Fabs). The final buffer used for all proteins is $20 \mathrm{mM}$ HEPES, $\mathrm{pH} 7.2$, and $150 \mathrm{mM} \mathrm{NaCl}$.

\section{Cryo-EM data collection, processing, and structure building}

The samples for cryo-EM study were prepared essentially as previously described ${ }^{51,53}$. All EM grids were evacuated for 2 min and glow-discharged for $30 \mathrm{~s}$ using a plasma cleaner (Harrick PDC-32G-2). Four microliters of spike protein $(0.8 \mathrm{mg} / \mathrm{mL})$ was mixed with the same volume of Fabs $(1 \mathrm{mg} / \mathrm{mL}$ each $)$, and the mixture was immediately applied to glow-discharged holy-carbon gold grids (Quantifoil, R1.2/1.3) in an FEI Vitrobot IV ( $4{ }^{\circ} \mathrm{C}$ and $100 \%$ humidity). Data collection was performed using either a Titan Krios G3 equipped with a K3 direct detection camera, or a Titan Krios G2 with a $\mathrm{K} 2$ camera, both operating at $300 \mathrm{kV}$. Data processing was carried out using cryoSPARC ${ }^{54}$. After 2D classification, particles with good qualities were selected for global 3D reconstruction and then subjected to homogeneous refinement. To improve the density surrounding the RBD-Fab region, UCSF Chimera ${ }^{55}$ and Relion ${ }^{56}$ were used to generate the masks, and local refinement was then performed using cryoSPARC. $\operatorname{Coot}^{57}$ and Phenix ${ }^{58}$ were used for structural modeling and refinement. Figures were prepared using USCF ChimeraX ${ }^{59}$ and Pymol (Schrödinger, LLC.). 


\section{Reference}

650

50 Ehrenmann, F. \& Lefranc, M. P. IMGT/DomainGapAlign: IMGT standardized analysis of amino acid sequences of variable, constant, and groove domains (IG, TR, MH, IgSF, MhSF). Cold Spring Harb Protoc 2011, 737-749, doi:10.1101/pdb.prot5636 (2011).

51 Du, S. et al. Structurally Resolved SARS-CoV-2 Antibody Shows High Efficacy in Severely Infected Hamsters and Provides a Potent Cocktail Pairing Strategy. Cell 183, 1013-1023 e1013, doi:10.1016/j.cell.2020.09.035 (2020).

52 Gui, M. et al. Cryo-electron microscopy structures of the SARS-CoV spike glycoprotein reveal a prerequisite conformational state for receptor binding. Cell Res 27, 119-129, doi:10.1038/cr.2016.152 (2017).

53 Du, S. et al. Structures of SARS-CoV-2 B.1.351 neutralizing antibodies provide insights into

54 Punjani, A., Rubinstein, J. L., Fleet, D. J. \& Brubaker, M. A. cryoSPARC: algorithms for rapid unsupervised cryo-EM structure determination. Nat Methods 14, 290-296, doi:10.1038/nmeth.4169 (2017). cocktail design against concerning variants. Cell research, doi:10.1038/s41422-021-00555-0 Pettersen, E. F. et al. UCSF Chimera--a visualization system for exploratory research and analysis. J Comput Chem 25, 1605-1612, doi:10.1002/jcc.20084 (2004).

\section{Declaration of interests}

X.S.X. and Y.C. are listed as inventors on the provisional patent applications of BD series antibodies. X.S.X. and Y.C. are founders of Singlomics Biopharmaceuticals. The remaining authors declare no competing interests.

\section{Corresponding authors}

Correspondence to Yunlong Cao or Ronghua Jin or Junyu Xiao or Xiaoliang Sunney Xie. Request for materials described in this study should be directed to Yunlong Cao and Xiaoliang Sunney Xie. 


\section{Data availability}

689

Logo plots of escape maps for antibodies in this study are available in Supplementary Data 1. Processed mutation escape scores can be downloaded at https://github.com/jianfcpku/SARS-CoV-2-RBD-DMS-broad. Raw Illumina and PacBio sequencing data are available on NCBI Sequence Read Archive BioProject PRJNA787091. We used vdj_GRCh38_alts_ensembl-5.0.0 as the reference of V(D)J alignment, which can be obtained from https://support.10xgenomics.com/single-cell$\mathrm{vdj} / \mathrm{software} /$ downloads/latest. IMGT/DomainGapAlign is based on the built-in lastest IMGT antibody database, and we let the "Species" parameter as "Homo sapiens" while kept the others as default. Public deep mutational scanning datasets involved in the study from literature could be downloaded at https://media.githubusercontent.com/media/jbloomlab/SARS2_RBD_Ab escape map s/main/processed data/escape data.csv.

\section{Code availability}

Codes for analyzing SARS-CoV-2 escaping mutation profile data are available at https:/github.com/sunneyxielab/SARS-CoV-2-RBD-Abs-HTDMS. R and Python scripts for reproducing figures in this manuscript are available at https://github.com/jianfcpku/SARS-CoV-2-RBD-DMS-broad.

.


bioRxiv preprint doi: https://doi.org/10.1101/2022.02.07.479349; this version posted February 7, 2022. The copyright holder for this preprint (which was not certified by peer review) is the author/funder, who has granted bioRxiv a license to display the preprint in perpetuity. It is made available under aCC-BY-NC-ND 4.0 International license.
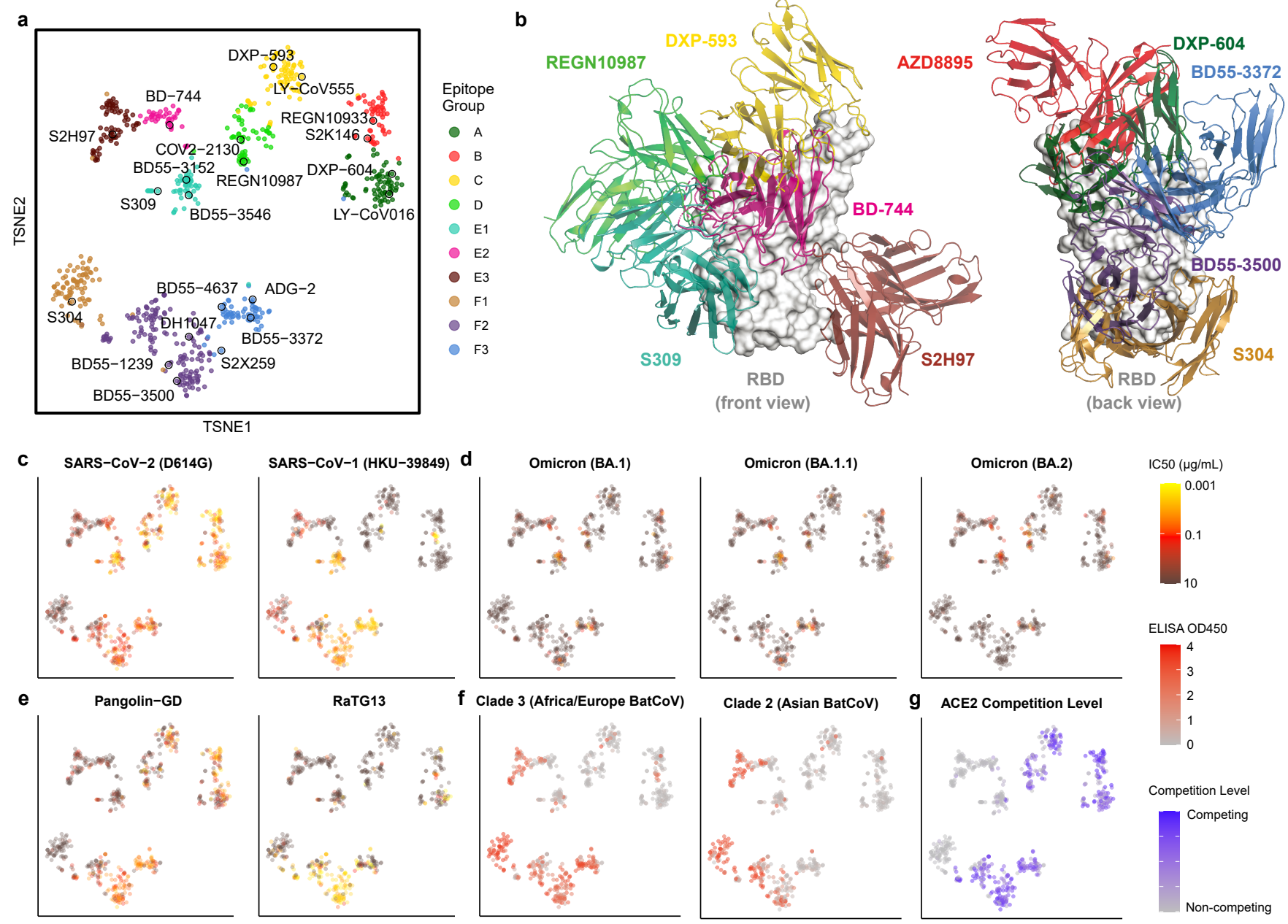

f Clade 3 (Africa/Europe BatCoV)
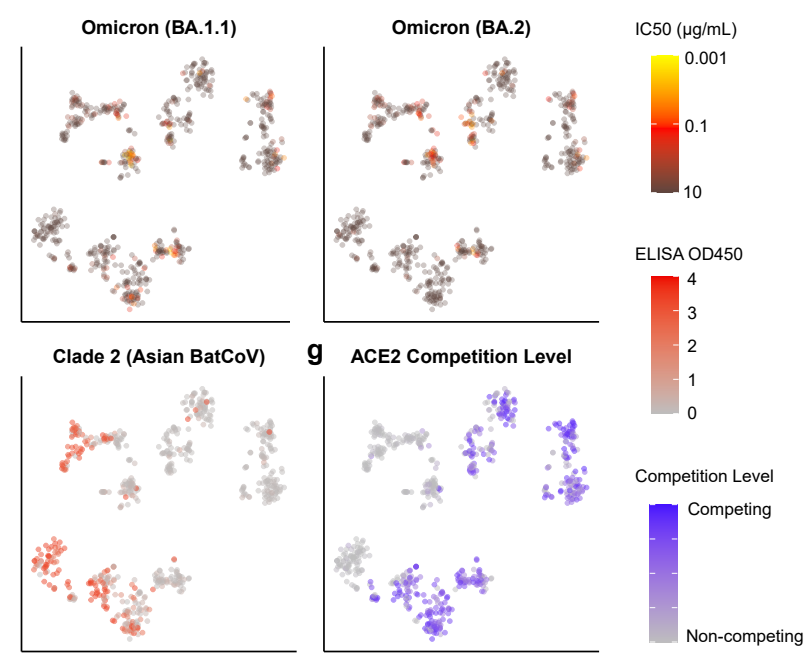

Fig. 1 | Comprehensive epitope and functional mapping of broad sarbecovirus neutralizing antibodies.

a, t-distributed stochastic neighbor embedding (t-SNE) and unsupervised k-means clustering of neutralizing antibodies against SARS-CoV-2 based on antibody escape maps characterized by MACS-based high-throughput deep mutational screening. b, Representative antibody structures of ten epitope groups. c-e, Neutralization of sarbecovirus (spike-pseudotyped VSV) by 715 RBD antibodies. Color bars indicate IC50 values $(\mu \mathrm{g} / \mathrm{mL})$. c, SARS-CoV-2 D614G and SARS-CoV-1 HKU-39849. d, Omicron variants BA.1, BA.1.1 and BA.2. e, SARS-CoV-2 related sarbecovirus Pangolin-GD and RaTG13. f, ELISA reactivity to various sarbecovirus RBD of different clades of 715 RBD antibodies. ELISA OD450 is measured using $0.3 \mu \mathrm{g} / \mathrm{mL}$ antigen and $1 \mu \mathrm{g} / \mathrm{mL}$ antibody. Shades of red show the average OD450 of BM48-31 and BtKY72 (for clade 3, left), and the average of YN2013, Shaanxi2011, SC2018, Rp3, ZXC21, ZC45 and Anlong112 (for clade 2, right). g, The ACE2 blocking activity for 715 RBD antibodies. Shades of blue show the competition level measured through competing ELISA. All pseudovirus neutralization assays and ELISA measure- 
bioRxiv preprint doi: https://doi.org/10.1101/2022.02.07.479349; this version posted February 7, 2022. The copyright holder for this preprint (which was not certified by peer review) is the author/funder, who has granted bioRxiv a license to display the preprint in perpetuity. It is made available under aCC-BY-NC-ND 4.0 International license.
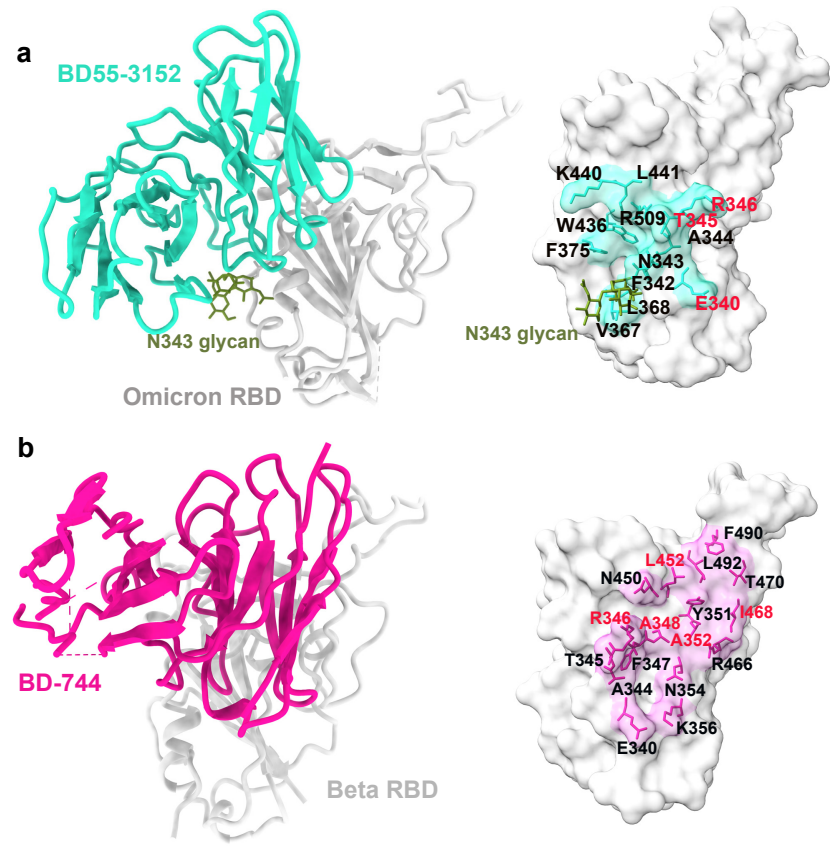

c

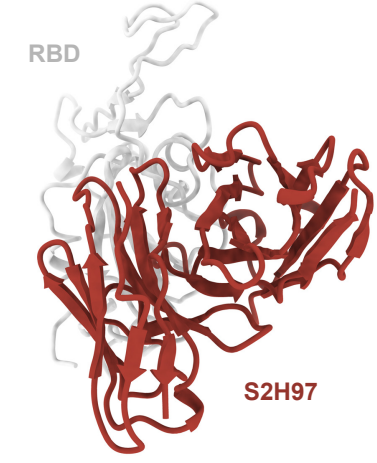

d

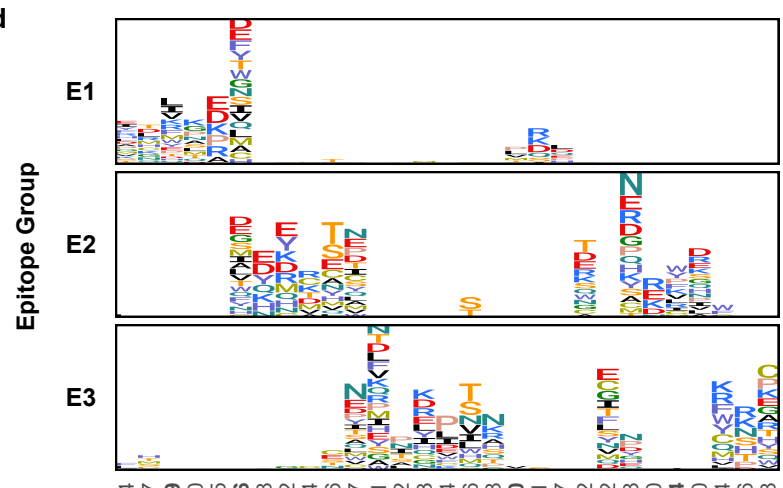

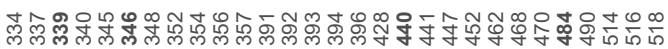

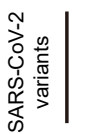

WT NPGETRAANKRCFTNYDNLGLKITEFSEL BA.1 NPDETRAANKRCFTNYDKLGLKITAFSEL BA.1.1 NPDETKAANKRCFTNYDKLGLKITAFSEL ? BA.2 NPDETRAANKRCFTNYDKLGLKITAFSEL RaTG13 NDGE RAANKR NYD Pangolin-GD NPGETTAANKRCFTNYDNLGLKI TEFSE Pangolin-CX NPGESKAANKRCFTNYDKQGLKI TVYSEL Oे Urbani NPGETKPAEKKCFSNYDNIGKR INPWSEL

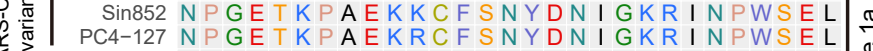
WIV1 NPGETTPAEKRCFSNYDNIGKRIN I WSEL LYRa11 NPGETTPAEKRCFSNYDN I GKR I NPWSEL LYRa11 NPGE A

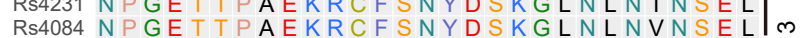

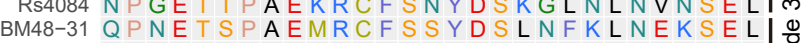
BAKY72 NPGQSNPAELRCFSSYDSVNYKINSESEL ZXC21 NPHKTRPAETKCFTSYDKQ-FKLS-RSEL ZC45 NPHKTRPAETKCFTSYDKQ-FKLS-RSEL HeB2013 NPDKTRPAETKCFTSYDKQ-FKLS-RSEL Rf4092 NPDATRPAEVKCFTSYDKQ-FKLS-RSEL YN2013 NPDS SRPAETKCFTSYDNQ-FKLS-RSEL As6526 NPDKTRPAETKCFTSYDQQ-YKLS-RSELL Rp3 NPDKTRPAETKCFTSYDKQ-YKLS-RSEL Shaanxi2011 NPDKTRPAETKCFTSYDNQ-YKLS-YSEL Yunnan2011 NP DRSRPAETKCFTS YENQ-YKLS-RSEL Rs4247 NPDKSRPAETKCFTSYDKQ-YKLS-YSEL K Longquan140 NPDKTRPAETKCFTSYDKQ-YKLS-YSEL

Fig. 2 | Structural and escaping mutation analyses of group E1-E3 antibodies.

a-c, High-resolution cryo-electron microscopy antibody structures of representative epitope group E1-E3 neutralizing antibodies. a, BD55-3152 (group E1) in complex of SARS-CoV-2 Omicron RBD complex. b, BD-744 (group E2) in complex of SARS-CoV-2 Beta RBD complex (PDB: 7EY0). c, S2H97 (group E3) in complex of SARS-CoV-2 RBD complex (PDB: $7 \mathrm{M} 7 \mathrm{~W}$ ). Residues on the binding interface are marked. Residues highlighted in red indicate featuring escaping hotspots of the representative epitope groups. $\mathbf{d}$, Averaged escape maps of antibodies in epitope group E1-E3, and corresponding multiple sequence alignment (MSA) of various sarbecovirus RBDs. Height of each amino acid in the escape maps represents its mutation escape score. Residues are colored corresponding to their chemical properties. Mutated sites in Omicron variants are marked in bold. 

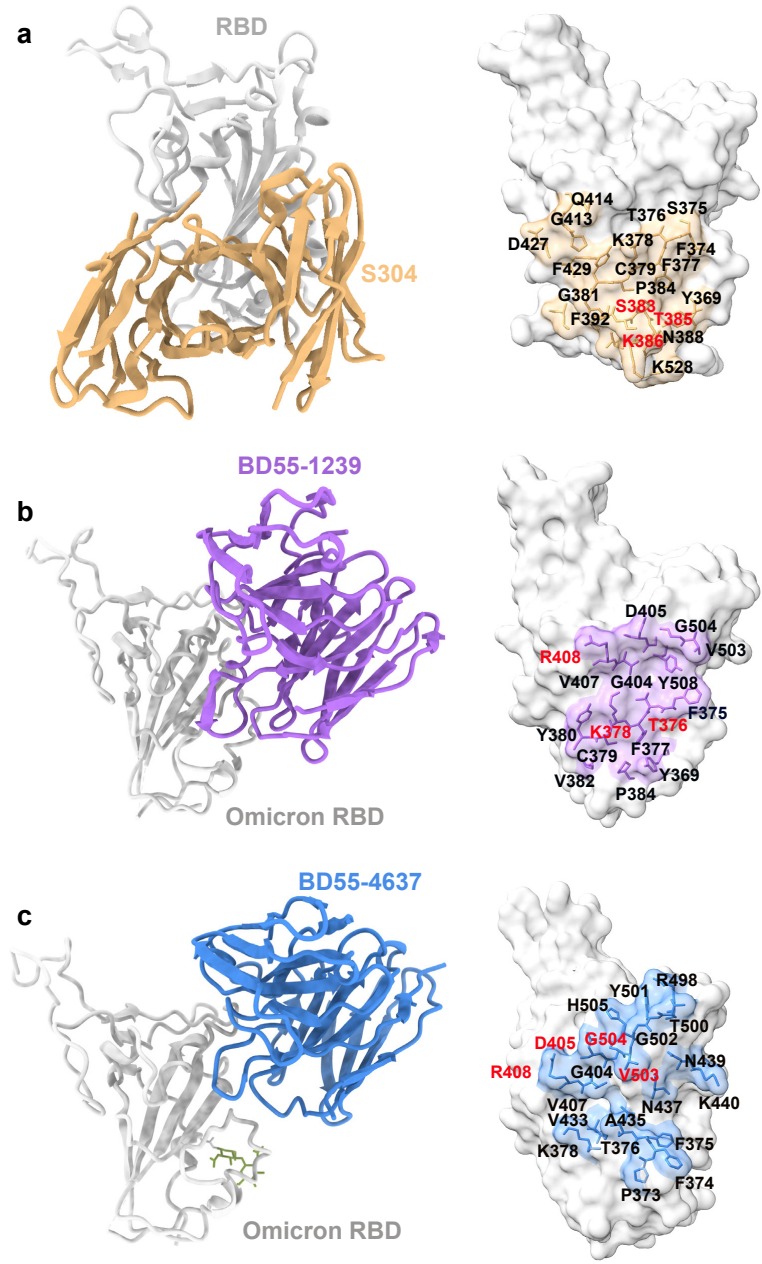

d
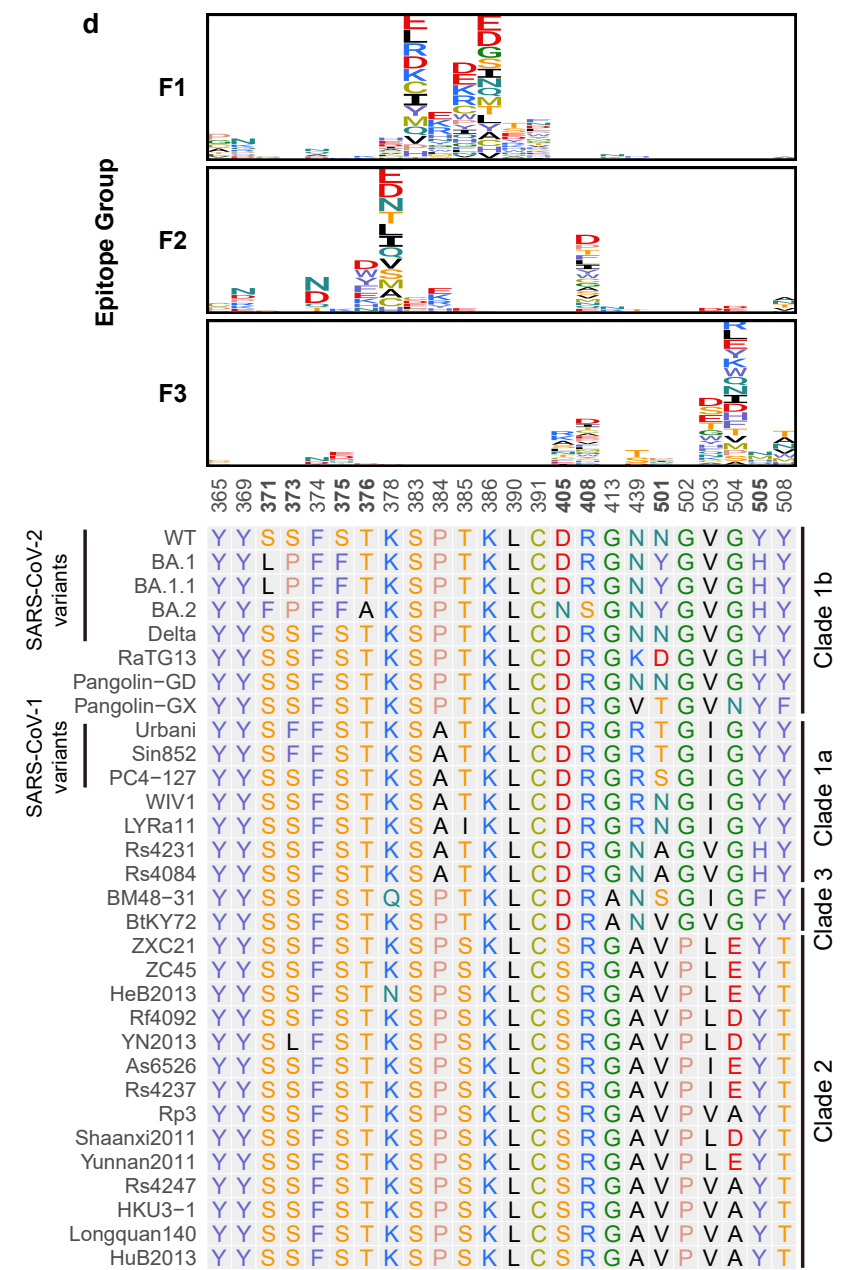

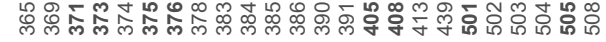

Fig. 3 | Structural and escaping mutation analyses of group F1-F3 antibodies.

a-c, High-resolution cryo-electron microscopy antibody structures of representative epitope group F1-F3 neutralizing antibodies. a, S304 (group F1) in complex of SARS-CoV-2 RBD complex (PDB: 7JW0). b, BD55-1239 (group F2) in complex of SARS-CoV-2 Omicron RBD complex. c, BD55-4637 (group F3) in complex of SARS-CoV-2 Omicron RBD complex. Residues on the binding interface are marked. Residues highlighted in red indicate featuring escaping hotspots of the representative epitope groups. d, Averaged escape maps of antibodies in epitope group F1-F3, and corresponding multiple sequence alignment (MSA) of various sarbecovirus RBDs. Height of each amino acid in the escape maps represents its mutation escape score. Residues are colored corresponding to their chemical properties. Mutated sites in Omicron variants are marked in bold. 


\begin{tabular}{|c|c|c|c|c|c|c|c|c|c|c|c|c|c|c|c|c|c|c|c|}
\hline \multicolumn{3}{|c|}{ Antibody } & S309 & $\begin{array}{c}\text { BD55- } \\
5840\end{array}$ & $\begin{array}{c}\text { BD55- } \\
3546\end{array}$ & $\begin{array}{c}\text { BD55- } \\
5549\end{array}$ & \begin{tabular}{|c|} 
BD55- \\
5585
\end{tabular} & S2H97 & $\begin{array}{c}\text { BD55- } \\
5263\end{array}$ & $\begin{array}{c}\text { BD55- } \\
5640\end{array}$ & $\begin{array}{c}\text { BD55- } \\
5242\end{array}$ & $\begin{array}{c}\text { BD55- } \\
3500\end{array}$ & $\begin{array}{c}\text { BD55- } \\
5700\end{array}$ & S2X259 & $\begin{array}{c}\text { BD55- } \\
3372\end{array}$ & $\begin{array}{c}\text { BD55- } \\
5514\end{array}$ & $\begin{array}{c}\text { BD55- } \\
5483\end{array}$ & $\begin{array}{c}\text { BD55- } \\
5558\end{array}$ & ADG-2 \\
\hline \multicolumn{3}{|c|}{ Epitope Group } & E1 & E1 & E1 & E1 & E1 & E3 & E3 & F2 & F2 & F2 & F2 & F3 & F3 & F3 & F3 & F3 & F3 \\
\hline & \multirow{7}{*}{ Pseudovirus } & D614G & 78.3 & 0.9 & 1.1 & 1.1 & 2.1 & 571.7 & 244.2 & 14.3 & 15.4 & 105.3 & 73.3 & 125.2 & 6.8 & 10.7 & 13.8 & 15.7 & 13.4 \\
\hline & & SARS-CoV-1 & 31.3 & 5.6 & 4.6 & 24.3 & 6.5 & 6743.2 & 51.1 & 14.3 & 4.4 & 52.2 & 10.5 & 46.2 & 13.8 & 4.4 & 5.8 & 4.0 & 1.7 \\
\hline & & BA.1 & 355.7 & 4.4 & 5.8 & 26.5 & 13.2 & 2407.6 & \begin{tabular}{|l|}
1785.4 \\
\end{tabular} & 69.2 & 25.3 & 578.2 & 66.9 & 1847.0 & 20.3 & 1.7 & 6.5 & 16.0 & 1467.0 \\
\hline & & BA.1.1 & 314.0 & 4.5 & 3.5 & 14.1 & 4.8 & 2231.2 & \begin{tabular}{|l|}
1922.4 \\
\end{tabular} & 79.3 & 44.5 & 1218.8 & 92.7 & 2341.2 & 14.0 & 3.0 & 5.2 & 12.5 & 990.6 \\
\hline & & BA.2 & 944.4 & 16.1 & 35.9 & 57.6 & 163.8 & 2015.1 & \begin{tabular}{|l|}
1771.8 \\
\end{tabular} & * & * & * & * & * & 105.1 & 18.7 & 13.3 & 51.8 & * \\
\hline & & Pangolin-GD & * & 295.7 & 1860.0 & * & 2099.9 & 97.6 & 295.0 & 18.3 & 17.3 & 8.7 & 20.3 & 15.2 & 3.2 & 57.0 & 7.9 & 13.4 & 5.0 \\
\hline & & RaTG13 & * & * & ${ }^{*}$ & * & * & 834.2 & \begin{tabular}{|l|}
219.1 \\
\end{tabular} & 2.5 & 0.8 & 3.1 & 2.1 & 1.1 & * & 37.5 & * & 1.9 & * \\
\hline & \multirow{2}{*}{ Authentic virus } & Wuhan-Hu-1 & 78.1 & 12.3 & 13.8 & 58.0 & 15.5 & 1365.4 & 1088.2 & 55.2 & 124.0 & 44.9 & 210.3 & 456.2 & 22.4 & 62.0 & 26.3 & 55.2 & 40.6 \\
\hline & & Omicron & 928.8 & 22.4 & 52.6 & 116.1 & 89.7 & \begin{tabular}{|l|l|}
7071.1 \\
\end{tabular} & \begin{tabular}{|l|}
7937.0 \\
\end{tabular} & 196.9 & 359.0 & 6729.5 & 787.5 & * & 58.0 & 49.2 & 49.2 & 156.3 & 3715.0 \\
\hline
\end{tabular}

\begin{tabular}{|c|c|c|c|c|c|c|c|c|c|c|c|c|c|c|c|c|c|c|c|}
\hline \multirow{23}{*}{ 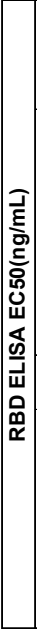 } & \multirow{4}{*}{$\begin{array}{c}\text { SARS-CoV-2 } \\
\text { clade(1b) }\end{array}$} & Wuhan-Hu-1 & 56.1 & 13.8 & 15.6 & 15.1 & 20.0 & 18.1 & 26.5 & 78.7 & 8.7 & 9.6 & 21.6 & 41.8 & 10.7 & 10.7 & 9.7 & 15.7 & 25.2 \\
\hline & & Pangolin-GD & 78.6 & 54.1 & 130.2 & $*$ & $*$ & 56.8 & 33.1 & 73.4 & 7.5 & 9.0 & 23.2 & 65.6 & 8.9 & 10.1 & 9.6 & 14.9 & 18.4 \\
\hline & & Pangolin-GX & 708.9 & * & * & * & * & 52.9 & 123.5 & 62.3 & 44.5 & 13.4 & 23.1 & * & * & * & * & * & * \\
\hline & & \begin{tabular}{|l|} 
RaTG13 \\
\end{tabular} & 144.7 & * & * & * & * & 41.2 & 129.2 & 66.3 & 27.1 & 15.6 & 24.7 & 61.2 & * & 35.4 & * & 58.3 & ${ }^{*}$ \\
\hline & \multirow{9}{*}{$\begin{array}{c}\text { SARS-CoV-1 } \\
\text { clade(1a) }\end{array}$} & BJ01 & 41.7 & 9.9 & 17.3 & 20.7 & 26.4 & 39.0 & 26.9 & 45.4 & 11.1 & 8.3 & 11.5 & 79.0 & 7.8 & 7.1 & 8.5 & 24.7 & 15.3 \\
\hline & & PC4-127 & 52.9 & 12.5 & 15.1 & 14.6 & 29.6 & 36.7 & 12.9 & 44.1 & 7.1 & 7.3 & 13.5 & 64.9 & 8.3 & 5.6 & 7.3 & 10.0 & 9.9 \\
\hline & & Sin852 & 71.4 & 16.4 & 29.2 & 28.8 & 43.5 & \begin{tabular}{|l|}
1627.4 \\
\end{tabular} & 15.2 & 47.5 & 12.5 & 11.2 & 14.9 & $*$ & 8.1 & 9.6 & 10.5 & 10.0 & 42.5 \\
\hline & & WIV1 & 57.7 & 32.8 & 247.3 & 272.9 & ${ }^{*}$ & 78.1 & 10.6 & 45.9 & 7.9 & 11.2 & 11.5 & 116.0 & 9.5 & 8.1 & 10.4 & 12.1 & 17.8 \\
\hline & & LYRa11 & 113.5 & 39.4 & 854.2 & $*$ & * & 91.4 & 51.7 & 64.6 & 21.1 & 19.2 & 18.0 & 98.9 & 19.1 & 11.3 & 15.5 & 32.8 & 24.6 \\
\hline & & Rs 7327 & 53.7 & 41.8 & 20.9 & 241.9 & * & 91.1 & 15.1 & 72.7 & 8.3 & 8.9 & 14.7 & 82.0 & 9.7 & 13.4 & 15.6 & 26.2 & 18.2 \\
\hline & & GZ-C & 116.2 & 13.6 & 43.5 & 33.6 & 34.2 & 34.3 & 23.7 & 52.5 & 14.2 & 13.7 & 13.2 & 97.3 & 15.4 & 10.3 & 12.1 & 18.1 & 17.0 \\
\hline & & Urbani & 139.3 & 33.4 & 464.6 & 58.7 & 91.1 & 1221.0 & 48.3 & 81.0 & 18.4 & 13.5 & 29.5 & 64.8 & 10.1 & 13.0 & 14.3 & 61.7 & 16.6 \\
\hline & & Rs4231 & 99.6 & * & * & $*$ & * & $*$ & 119.7 & 81.9 & 17.1 & 9.4 & 17.8 & 38.1 & 7.2 & 50.3 & 38.3 & 38.8 & 24.8 \\
\hline & \multirow{2}{*}{\begin{tabular}{|c|} 
Africa/Europe \\
BatCoV clade(3) \\
\end{tabular}} & BM48-31 & * & * & * & * & * & 64.7 & 585.4 & 190.5 & 86.8 & 40.7 & * & 82.9 & 55.9 & 27.3 & 17.5 & 118.4 & * \\
\hline & & BtKY72 & * & * & * & * & * & 75.3 & 60.9 & 57.6 & 14.7 & 12.6 & 21.2 & 77.1 & 14.0 & 13.8 & 12.9 & 27.0 & 52.9 \\
\hline & \multirow{8}{*}{$\begin{array}{c}\text { Asia BatCoV } \\
\text { clade(2) }\end{array}$} & Shaanxi2011 & * & * & * & * & * & 41.9 & 113.3 & 81.7 & 34.4 & 24.9 & 30.8 & * & $*$ & * & $*$ & * & $*$ \\
\hline & & \begin{tabular}{|l|} 
YN2013 \\
\end{tabular} & * & * & * & * & * & 44.2 & 39.5 & 83.1 & 40.1 & 21.7 & 66.4 & * & * & * & * & * & * \\
\hline & & Rp3 & * & * & * & * & * & 37.3 & 1.5 & 76.1 & 43.6 & 35.8 & 19.7 & * & * & * & * & * & * \\
\hline & & Rs4247 & * & * & * & * & * & 22.9 & 87.7 & $*$ & 31.7 & 26.4 & 30.5 & 323.8 & * & * & * & * & * \\
\hline & & ZC45 & * & * & * & * & * & 44.3 & 47.9 & 73.1 & 30.3 & 118.3 & 46.1 & * & * & * & * & * & * \\
\hline & & Anlong112 & * & * & * & * & * & 39.6 & 41.0 & 139.8 & 49.6 & 39.1 & 149.2 & * & * & * & * & * & * \\
\hline & & SC2018 & * & * & * & * & * & 42.0 & 44.8 & 174.6 & 28.2 & 15.7 & 60.4 & * & * & * & * & * & * \\
\hline & & ZXC21 & * & * & * & * & * & 24.4 & 72.6 & * & 28.9 & 51.8 & 63.4 & * & * & * & * & * & * \\
\hline
\end{tabular}

b

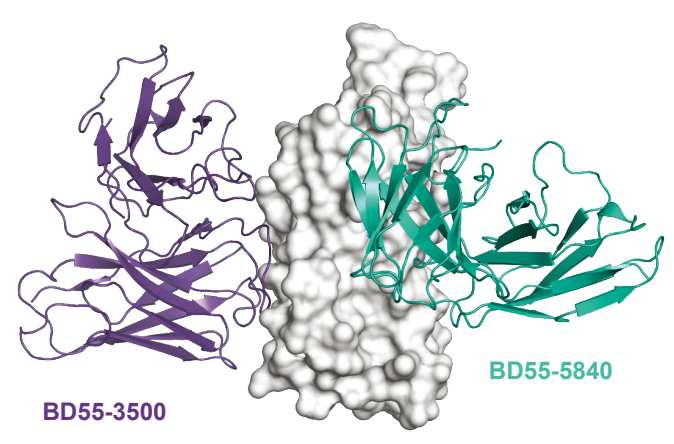

Omicron RBD

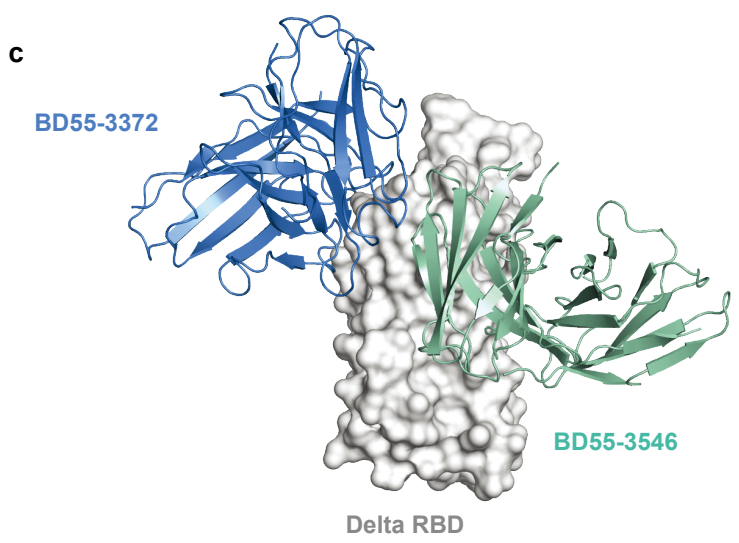

Fig. 4 | Potency and breadth of selected sarbecovirus neutralizing antibody drug candidates.

a, The sarbecovirus neutralization and binding capability (half-maximum effective concentration, EC50) of selected potent broad-spectrum neutralizing antibodies. *: $>10,000$ $\mathrm{ng} / \mathrm{mL}$ for IC50, or $>2,000 \mathrm{ng} / \mathrm{mL}$ for EC50. $\mathbf{b}$, Structural model of the non-competitive broad sarbecovirus neutralizing antibodies BD55-3500 (group F2, in complex of Omicron RBD) and BD55-5840 (group E1, in complex of Omicron RBD). c, Structural model of the non-competitive broad sarbecovirus neutralizing antibodies BD55-3372 (group F3, in complex of Delta RBD) and BD55-3546 (group E1, in complex of Delta RBD). 

perpetuity. It is made available under aCC-BY-NC-ND 4.0 International license.

a

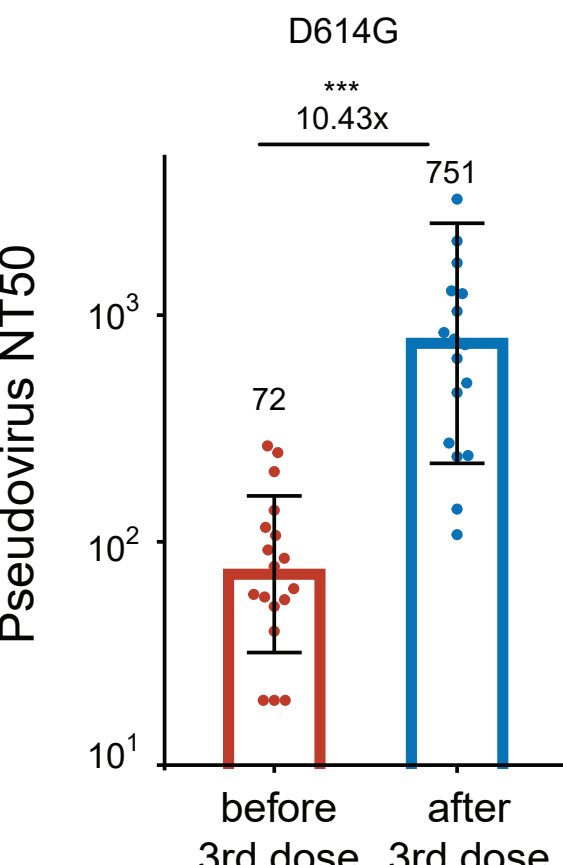

C

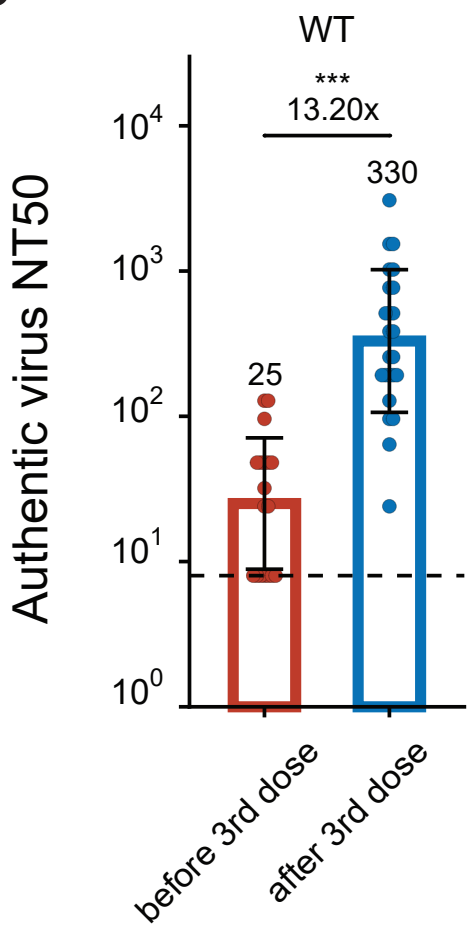

SARS
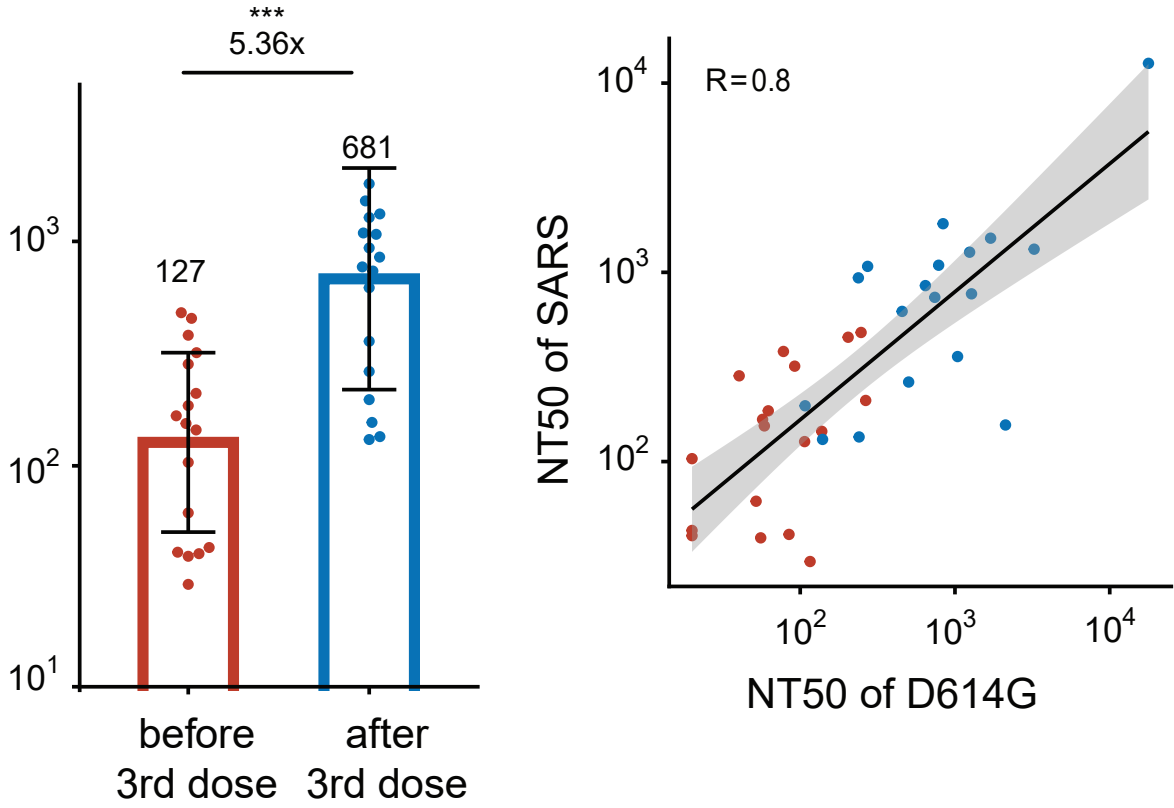

- before 3rd dose

- after 3rd dose

\section{Extended Data Fig. 1 | Neutralization titre of plasma specimens from SARS convalescents} vaccinated with 3 dose of SARS-CoV-2 vaccine.

a, Half-maximal neutralization titres (NT50) of plasma against SARS-CoV-2 with D614G mutant (D614G) and SARS-CoV-1 (SARS) pseudovirus, for SARS convalescents before and after the third dose vaccination $(n=18)$. b, Scatter plot showing the correlation between plasma NT50 against D614G pseudovirus and SARS pseudovirus for SARS convalescents before (red) and after (blue) the third dose vaccination $(n=18)$. Pearson's correlation coefficient is labeled. c, NT50 of plasma against different SARS-CoV-2 variants authentic virus, for SARS convalescents before and after the third dose vaccination $(n=18)$. Statistical significance in a, c was determined by two-tailed Wilcoxon signed rank test $\left({ }^{* * *} p<0.001,{ }^{* *} p<0.01,{ }^{*} p<0.05\right)$. NT50 values are displayed as geometric mean $\pm \mathrm{s}$. $\mathrm{d}$. in the $\log 10$ scale. 

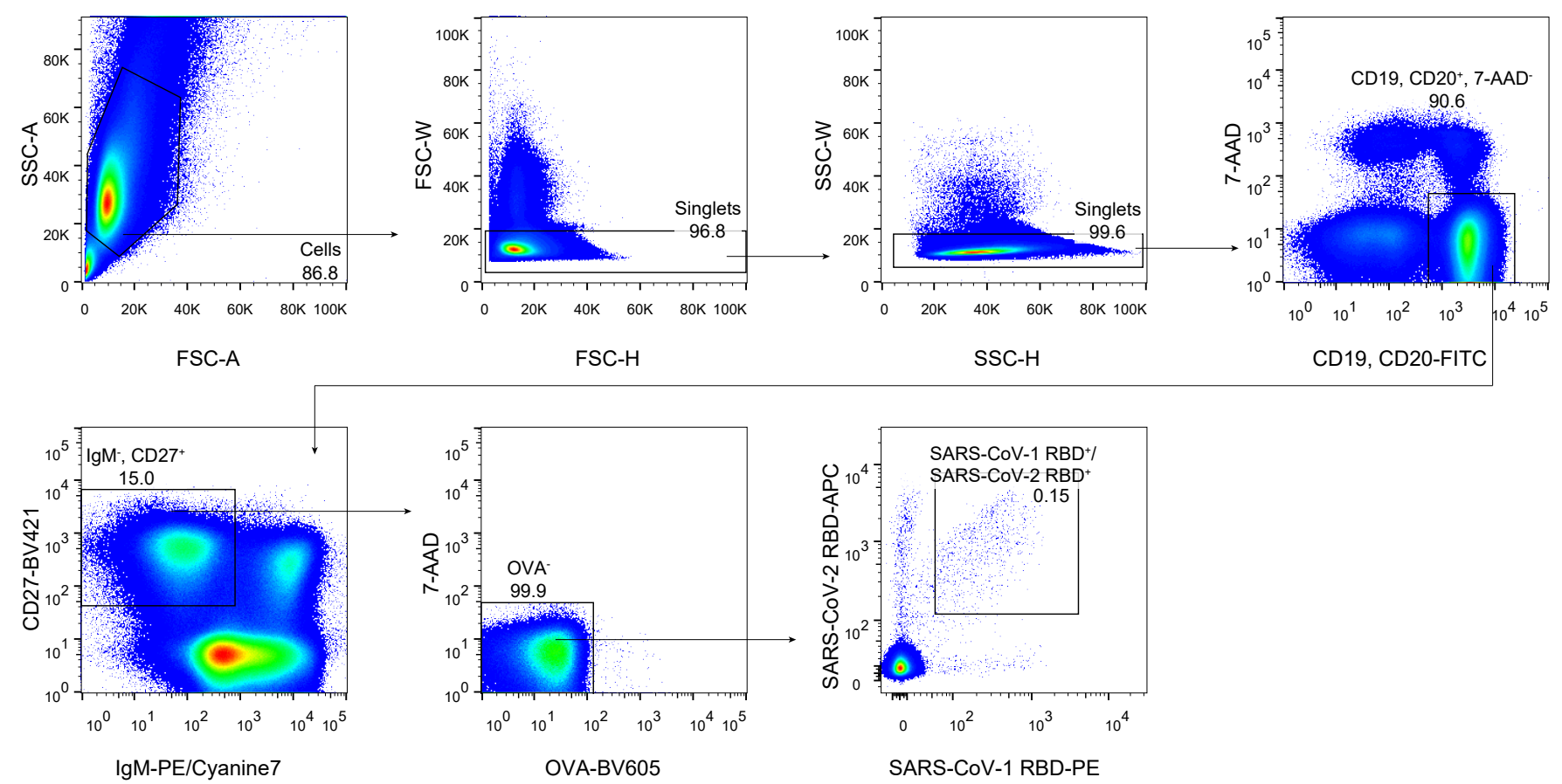

\section{Extended Data Fig. 2 | FACS of SARS-CoV-1 RBD and SARS-CoV-2 RBD cross-binding B cells. \\ The gating strategy for sorting SARS-CoV-1-RBD+/SARS-CoV-2-RBD+ single memory $B$ cells. Numbers next to outlined areas indicate percentage of cells in the gate. Sorting of the PBMCs from SARS convalescents that received 3 doses of the SARS-CoV-2 vaccine are shown.}


bioRxiv preprint doi: https://doi.org/10.1101/2022.02.07.479349; this version posted February 7, 2022. The copyright holder for this preprint (which was not certified by peer review) is the author/funder, who has granted bioRxiv a license to display the preprint in perpetuity. It is made available under aCC-BY-NC-ND 4.0 International license.

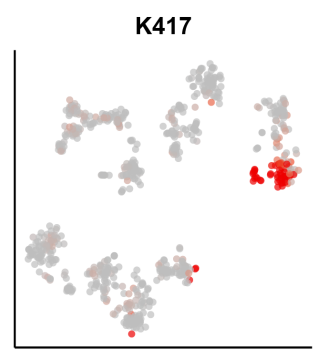

E484

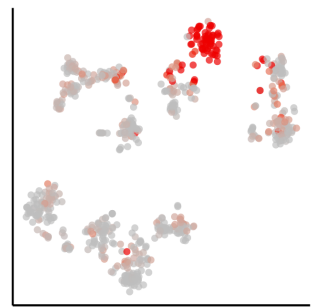

L452

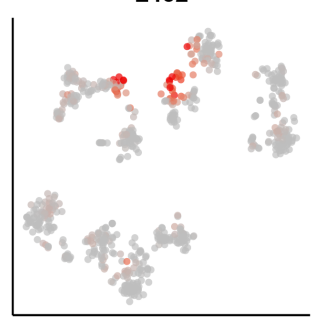

S514

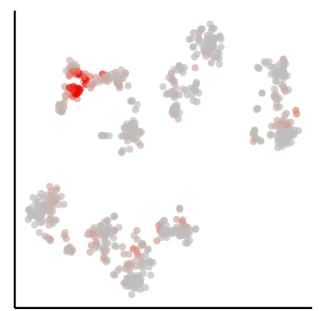

S383

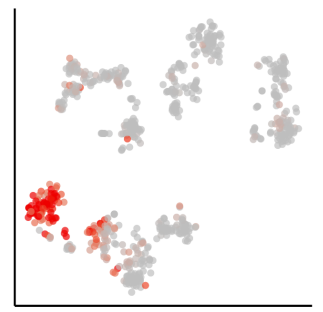

D405

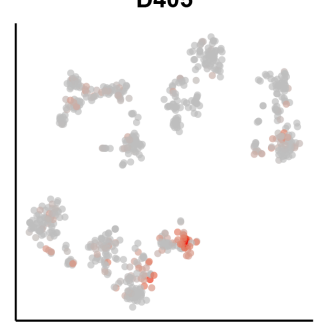

D420

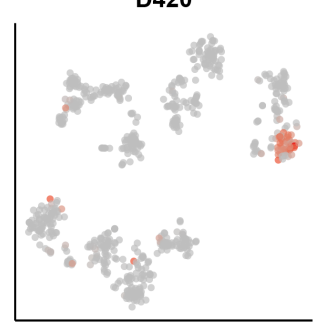

F490

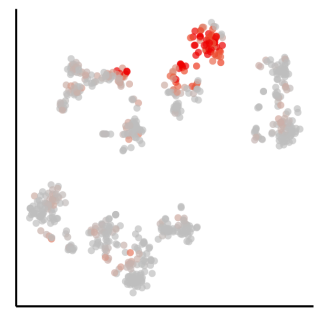

G339

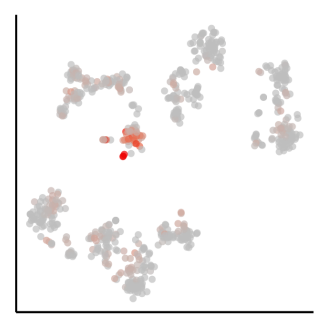

E516

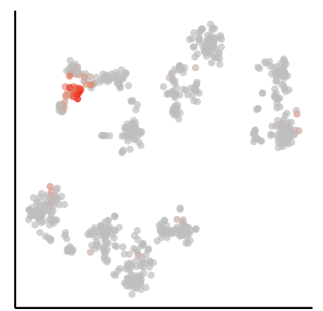

T385

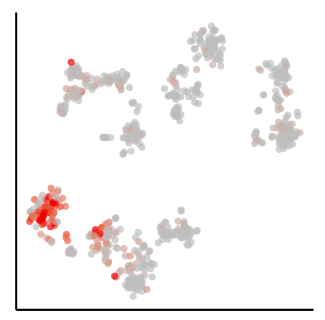

R408

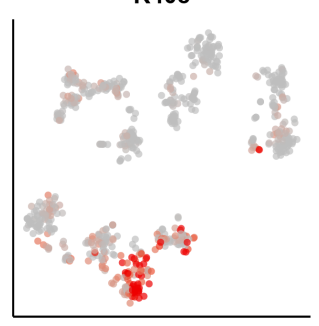

F456

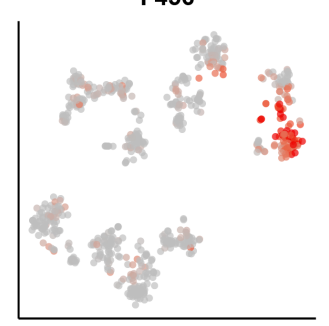

N440

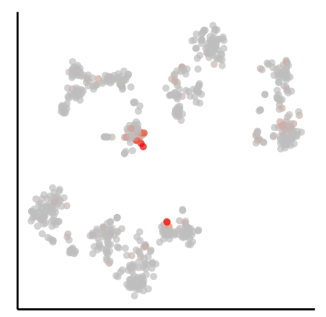

T345

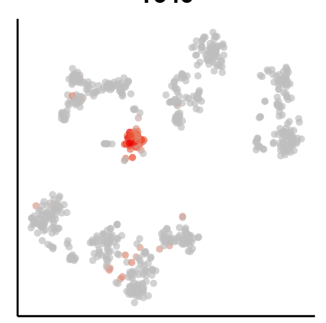

L518

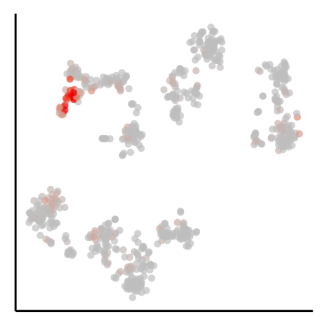

K386

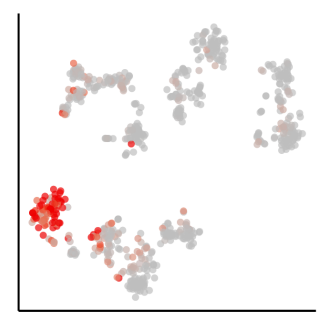

G504

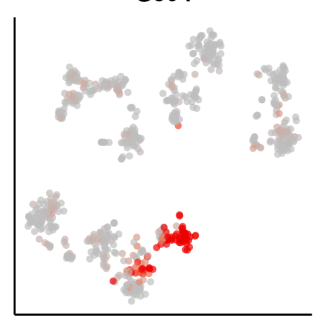

A475

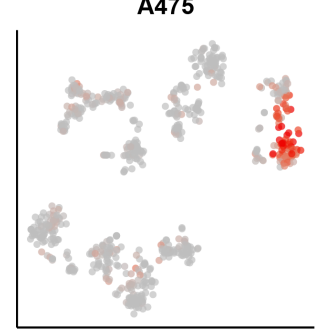

K444

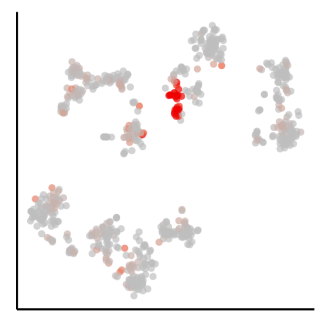

R346

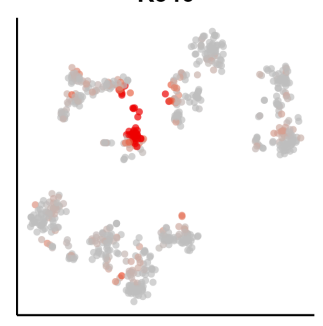

C391

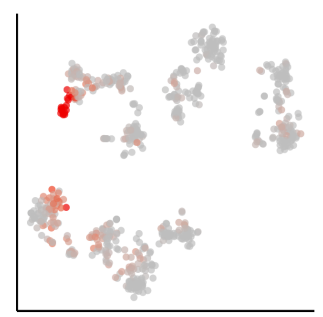

T376

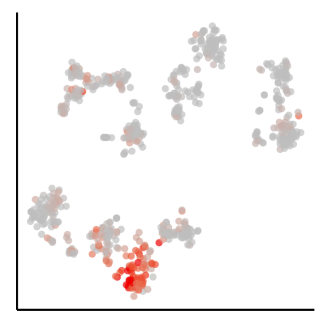

Y508

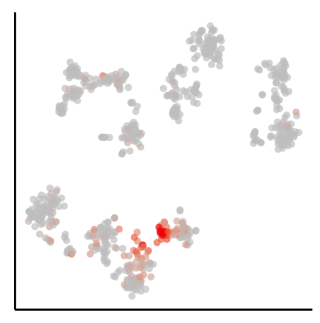

F486

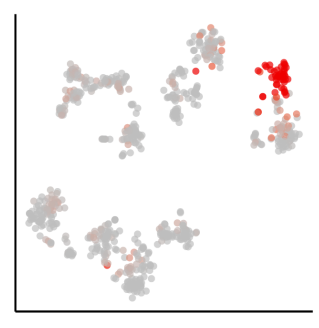

G446

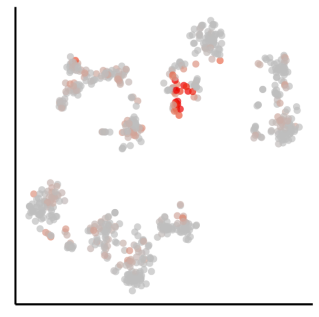

A348

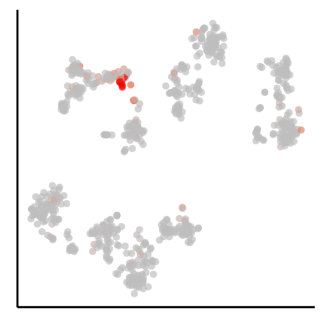

K462

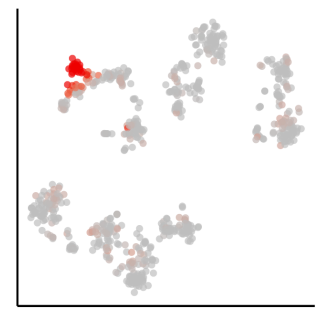

K378

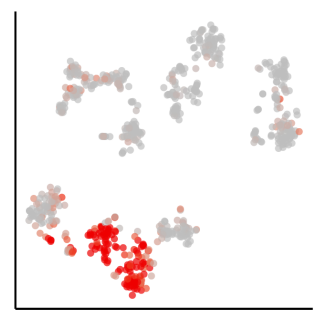

Normalized

Site Escape Score

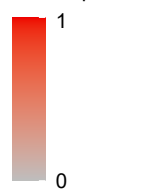

Extended Data Fig. 3 | Escape scores projection of top RBD escaping hotspots.

Shades of red indicate normalized site total escape scores of the representative residues for each antibody inferred from yeast display deep mutational screening. 
bioRxiv preprint doi: https://doi.org/10.1101/2022.02.07.479349; this version posted February 7, 2022. The copyright holder for this preprint (which was not certified by peer review) is the author/funder, who has granted bioRxiv a license to display the preprint in

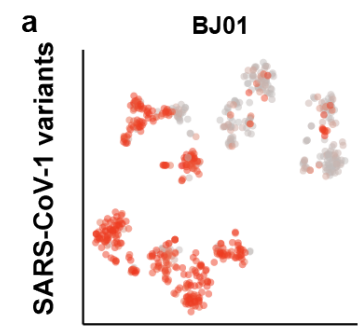
perpetuity. It is made available under aCC-BY-NC-ND 4.0 International license.
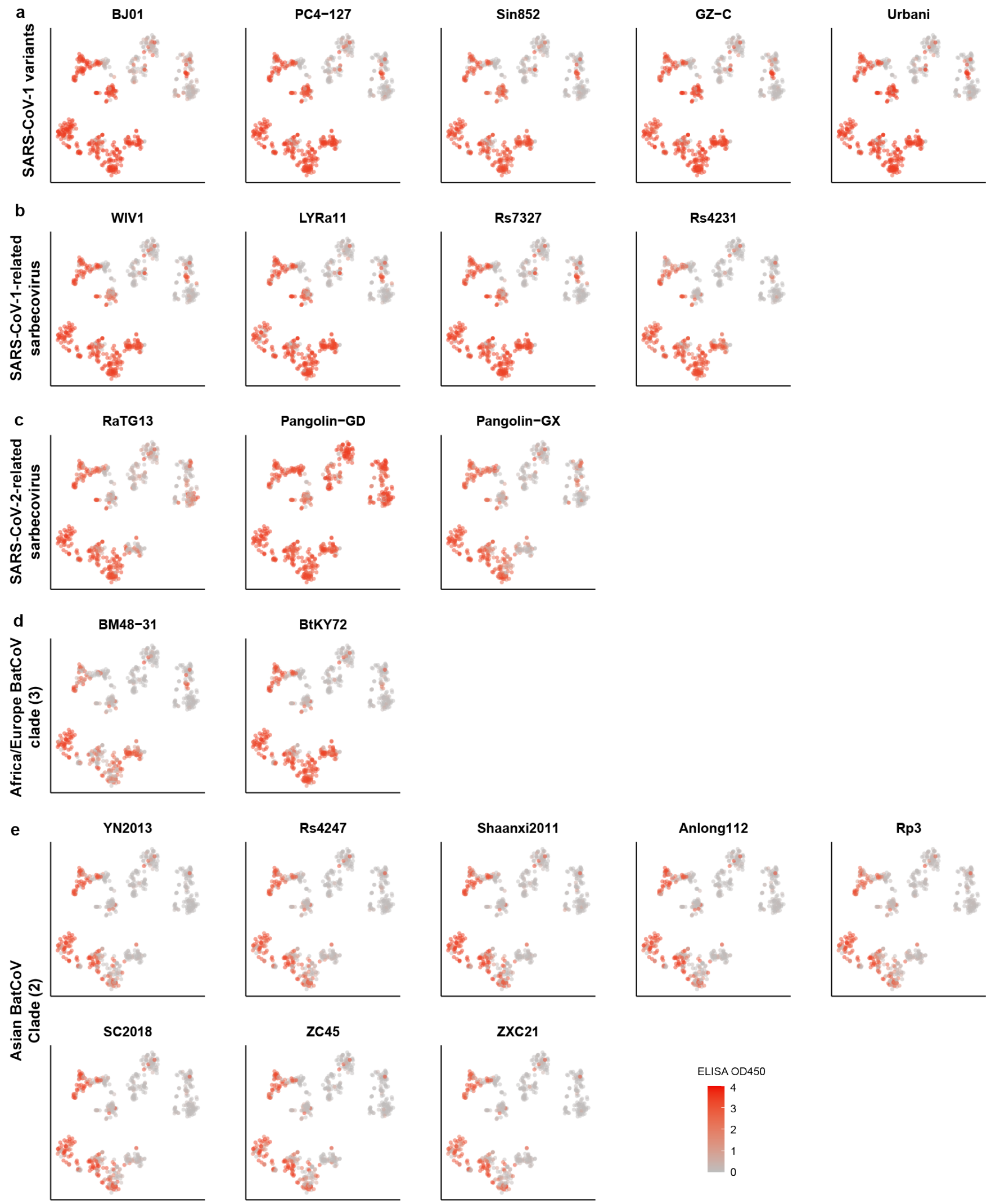

Extended Data Fig. 4 | Projection of ELISA reactivity against 22 sarbecovirus RBD.

a-e, Shades of red indicate ELISA OD450 for each antibody against various sarbecovirus clades. (a, SARS-CoV-1 variants. b, SARS-CoV-1-related sarbecovirus. c, SARS-CoV-2 related sarbecovirus. d, Africa/Europe batcoronavirus. e, Asian non-ACE2-utilizing batcoronavirus.) 
bioRxiv preprint doi: https://doi.org/10.1101/2022.02.07.479349; this version posted February 7, 2022. The copyright holder for this praprint (which was not certified by peer review) is the author/funder, who has granted bioRxiv a license to display the preprint in D614Rrpetuity. It is made available under aCC-BY-NC-ND 4.0 International license.

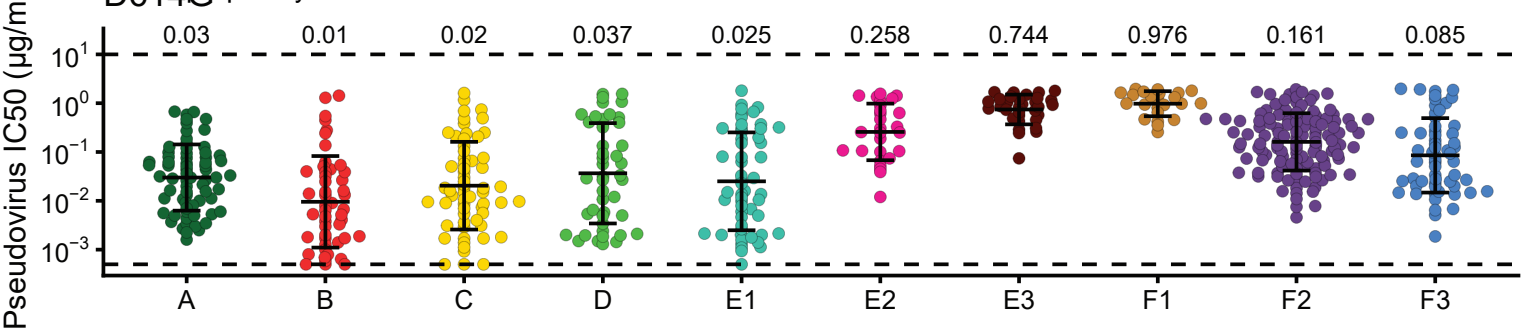

b

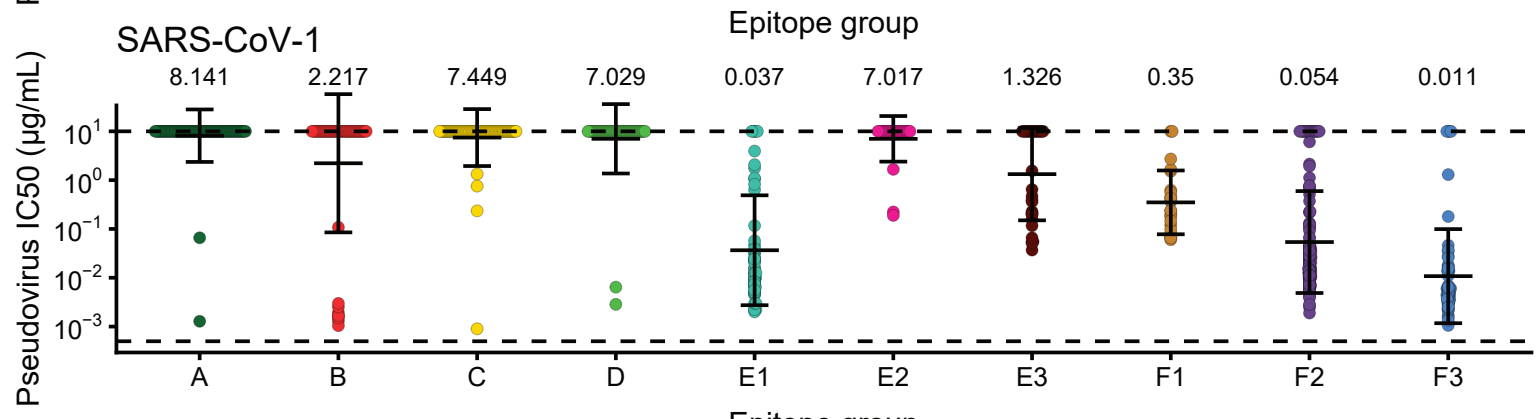

C

Omicron (BA.1)

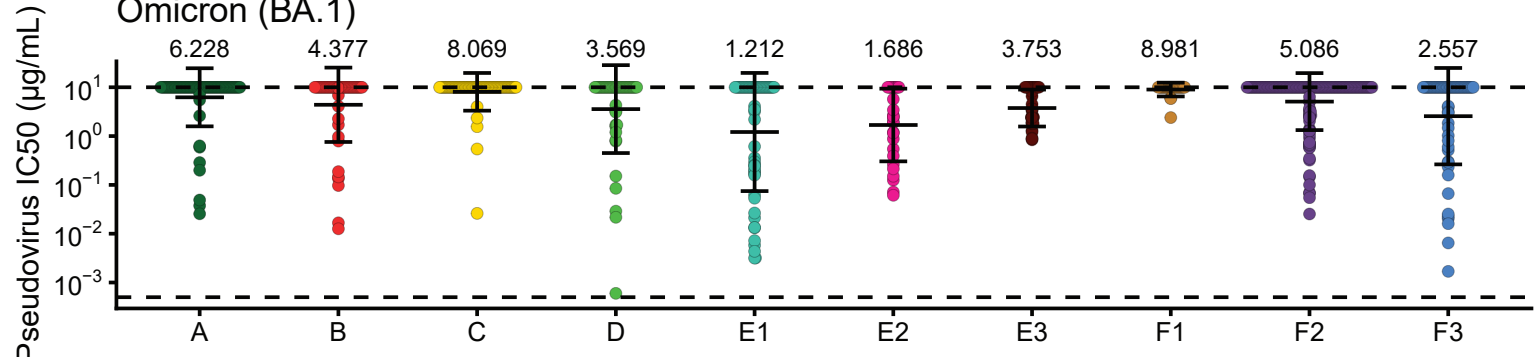

d

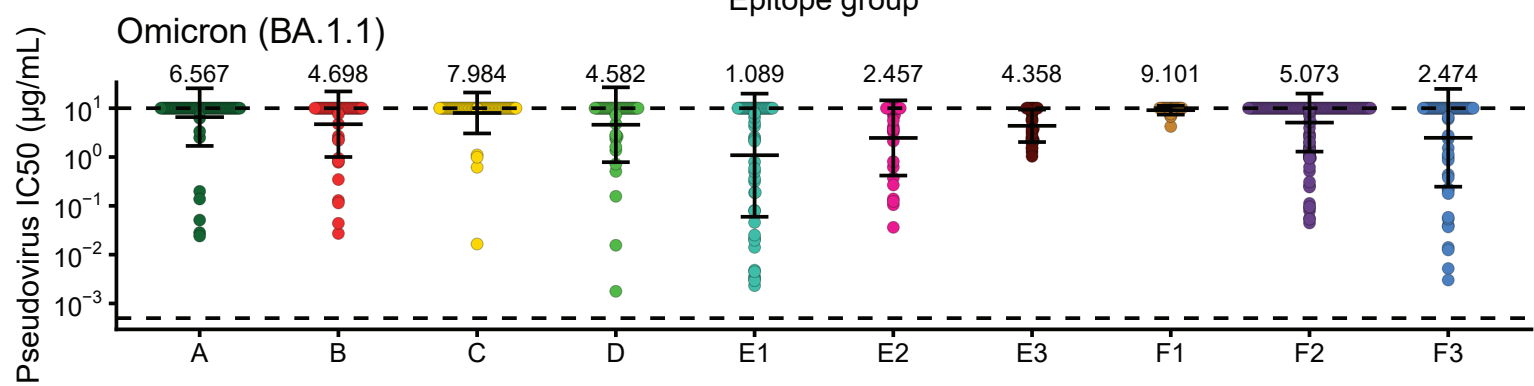

e

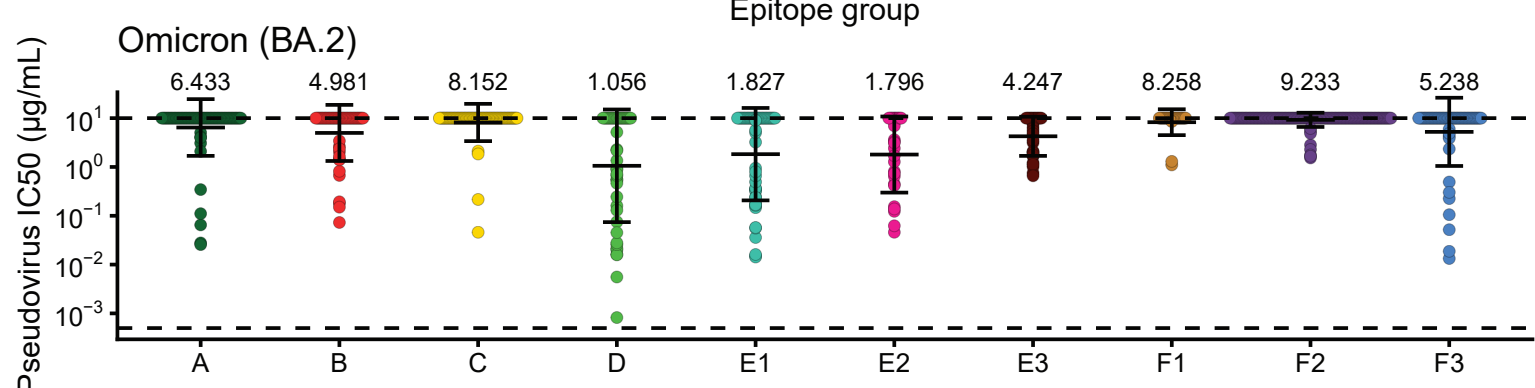

f

RaTG13

Epitope group

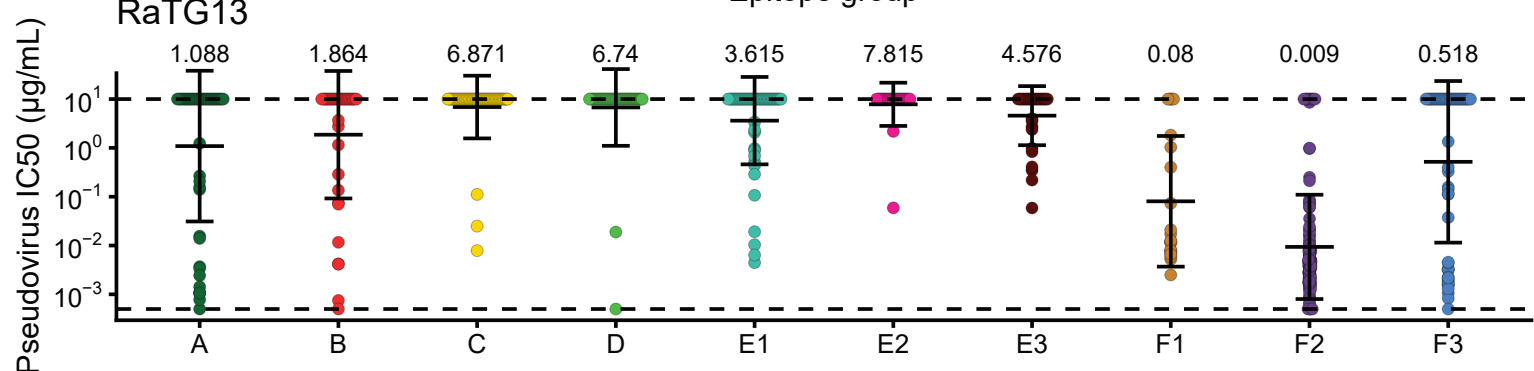

g

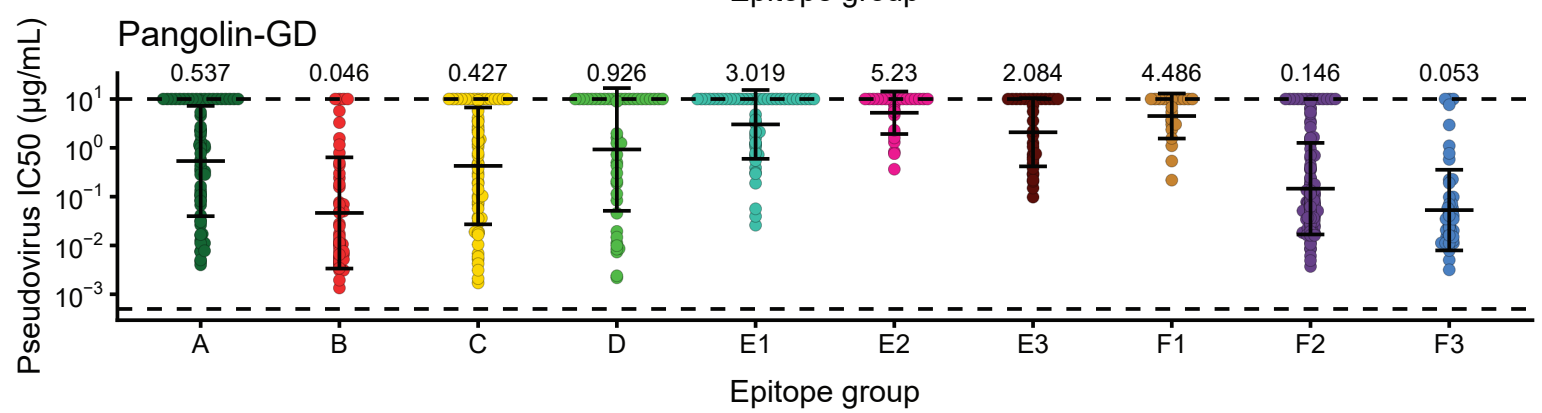




\section{Extended Data Fig. 5 | Neutralization potency for NAbs of each epitope group.}

a-g, Half maximal inhibitory concentration (IC50) of antibodies against different sarbecovirus determined by pseudovirus neutralization assay. The IC50 values are shown as geometric mean $\pm \mathrm{s}$. d. in $\log 10$ scale. Dashed lines show the detection limit, which is from $0.0005 \mu \mathrm{g} / \mathrm{mL}$ to $10 \mu \mathrm{g} / \mathrm{mL}$. Number of antibodies $\mathrm{n}=68,49,61,45,53,27,31,23$, 126, 57 for epitope group A, B, C, D, E1, E2, E3, F1, F2, F3, respectively (except for f). a, SARS-CoV-2 with D614G mutation. b, SARS-CoV-1 (HKU-39849). c, SARS-CoV-2 Omicron (BA.1). d, SARS-CoV-2 Omicron (BA.1.1). e, SARS-CoV-2 Omicron (BA.2). f, RaTG13, $\mathrm{n}=50,38,47,41,52,27,31,23,125,56$ for epitope group A, B, C, D, E1, E2, E3, F1, F2, F3, respectively. g, Pangolin-GD. 
a

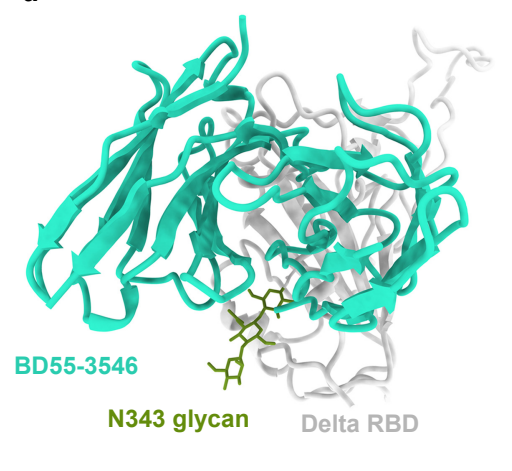

C

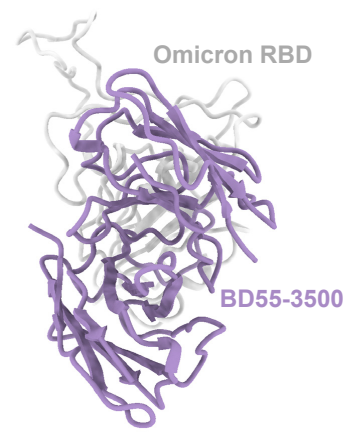

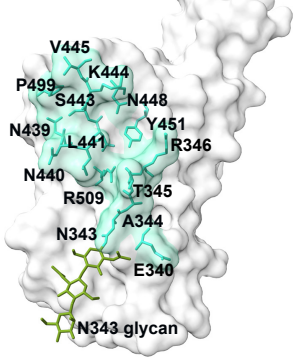

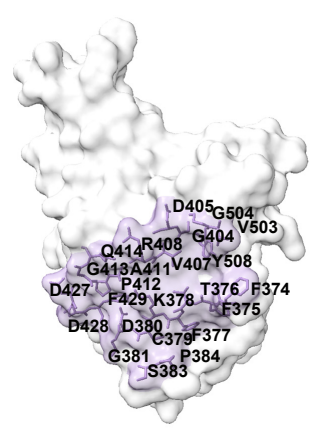

b

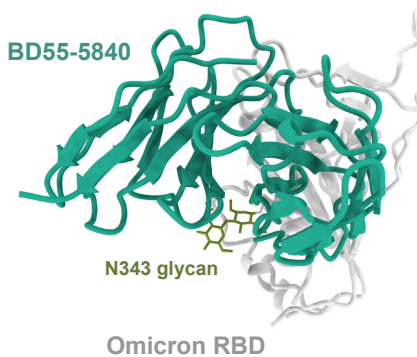

d

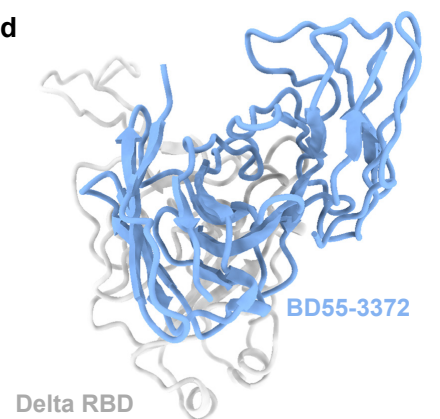

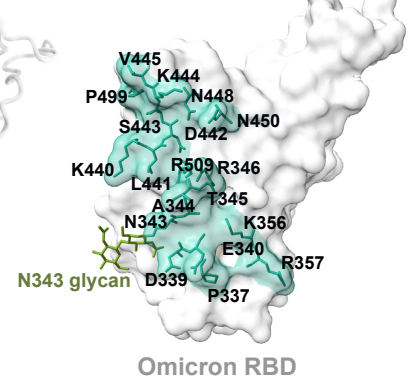

Omicron RBD

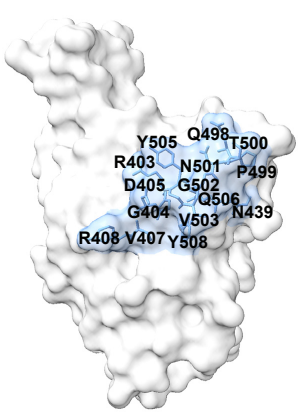

Extended Data Fig. 6 | Structures of individual broad sarbecovirus neutralizing antibody drug candidates.

a, BD55-3546 Fab in complex of Delta RBD. b, BD55-5840 Fab in complex of Omicron RBD. c, BD55-3500 Fab in complex of Omicron RBD. d, BD55-3372 Fab in complex of Delta RBD. Residues on the binding interface are marked. 
bioRxiv preprint doi: https://doi.org/10.1101/2022.02.07.479349; this version posted February 7, 2022. The copyright holder for this preprint (which was not certified by peer review) is the author/funder, who has granted bioRxiv a license to display the preprint in perpetuity. It is made available under aCC-BY-NC-ND 4.0 International license.

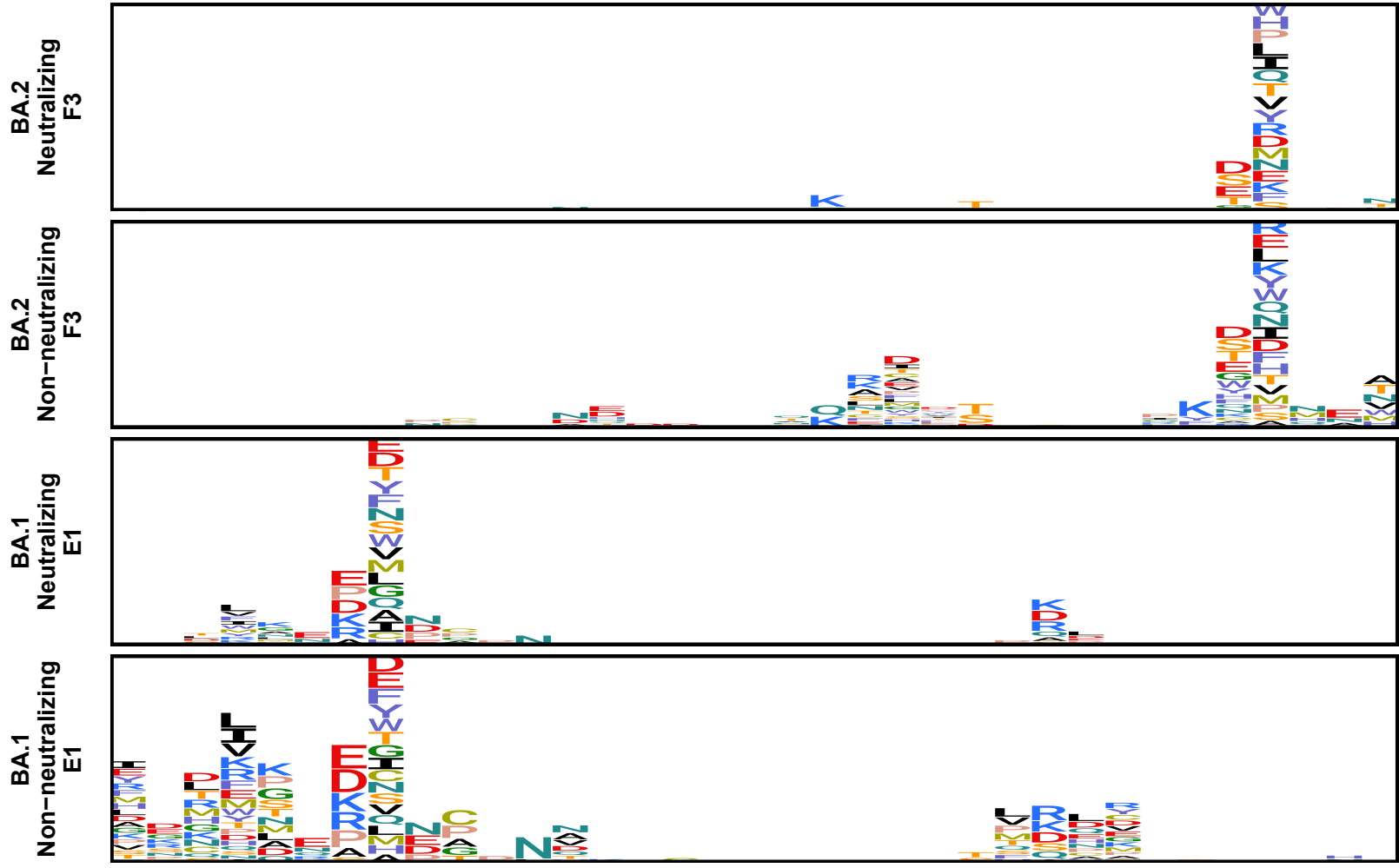

崩忿

WT N L P E A TR C Y Y F S T K SPR GDRKN N L N N G V G Y Y BA.1 N L P D A T R C Y Y L F F K S P R G R N N K G N Y G V G H Y BA.1.1 N L P D A T K C Y Y L F F K S P R G R N N K G N Y G G G Q Y BA.2 N L P D A T R C Y Y F F A K S P R G S N N L G N Y G V G $\mathrm{Q} Y$ Delta N L P E A TR C Y Y S F T K S P R D R K N L G N G G G Y Q Y RaTG13 N L P G E A T T C Y Y S S T K S P T G D R K K H I G N D G G H Y Pangolin-GD N L P G A T T C Y Y S F S T K PR GDR R N L G N G G G Y Q

Pangolin-GX N L P G A S K C Y Y S F S T K S P K G D R V K Q G N T G V Y Q F Urbani N L P G A T K C Y Y S F S T K S A K G R R R N I G N T I G Y Q Y BJ01 N L P G A TKC Y Y S F S T K S A K G D R R N I G N T G I G Y Q Y GZ-C N L P G A T K C Y Y S F S T K S K G D R V R N I G N T G I G Y Y $\operatorname{Sin} 852 N L P G E A T K C Y Y S F S T K S A K G D R V R N I G N T G I G Y Q Y$ PC4-127 N L P G E A T K Y Y S F S T K S A K G D R V R N I G N S G I G Y Q Y

WIV1 N L P G A T T C Y Y S F T K S A K G D R R N I G N N G I G Q Y Rs7327 N L P G A T T C Y Y S F T K S A K G D R R N I G N N G G Y Q Y LYRa11 N L P G A T T C Y Y S F S T K A K G D R V R N I G N N G G Y Q Y Rs4231 N L P G A T T C Y Y S F S T K S A K D R V N S K G N A G G H Q BM48-31 Q L P N E I T S C Y Y S F T Q S P K G R V N S L N E S I G F Q Y BtKY72 N L P G Q A N C Y Y S F S T K S K G D R V N S V N V G V G $\mathrm{Y} Y$ ZXC21 NVPHKATR C Y Y F S TKSPRF SRVAKQ - - VPLEYQT ZC45 NVPHKATR C Y Y F S T K SPRFSRVAKQ - - VPLEYQT Anlong112 NRP D S A S R Y Y S F T K S P R S R V A N Q - V V L D Y Q T Rf4092 N L P D A A T R Y Y S F S TKSPR F S R A K Q - - VPLDYQT YN2013 N R P D S A S R Y Y S F S T K S P R SR V A N Q - - VPLDYQT SC2018 NRP D K A S R C Y Y S F S T K S P R S R R A K Q - - VPVA Y Q T Rs4237 NRP D K A S R C Y Y S F T K S P R S S R V A K Q - - VPI E Y Q T

Rp3 NRPDKA TR C Y Y S F T K SPR S S R A K Q - - VPVAYQT Shaanxi2011 NRP D K A T R C Y Y S F S T K S P R S S R V A N Q - - VPLDYQT Yunnan2011 NR P D R A S R C Y Y S F S T K S P R F S R V A N Q - V V L E Y Q T Rs4247 NRP D K A S R C Y Y S F TKSPR S R R A K Q - - V P V A Y Q T HKU3-1 NRP DKA TR C Y Y S F S T K S P R S S R V A K H - - VPVAYQT Longquan140 NR P D K V TR C Y Y S F T K S P R S R V A K Q - - V P V A Y T HuB2013 NRP D R A S R C Y Y F S T K S P R S R V A K Q - - VPVAYQT antibodies in epitope group E1 and F3.

Height of each amino acid in the escape maps represents its mutation escape score. Residues are colored corresponding to their chemical properties. Mutated sites in Omicron variants (including BA.1, BA.1.1 and BA.2) are marked in bold. 


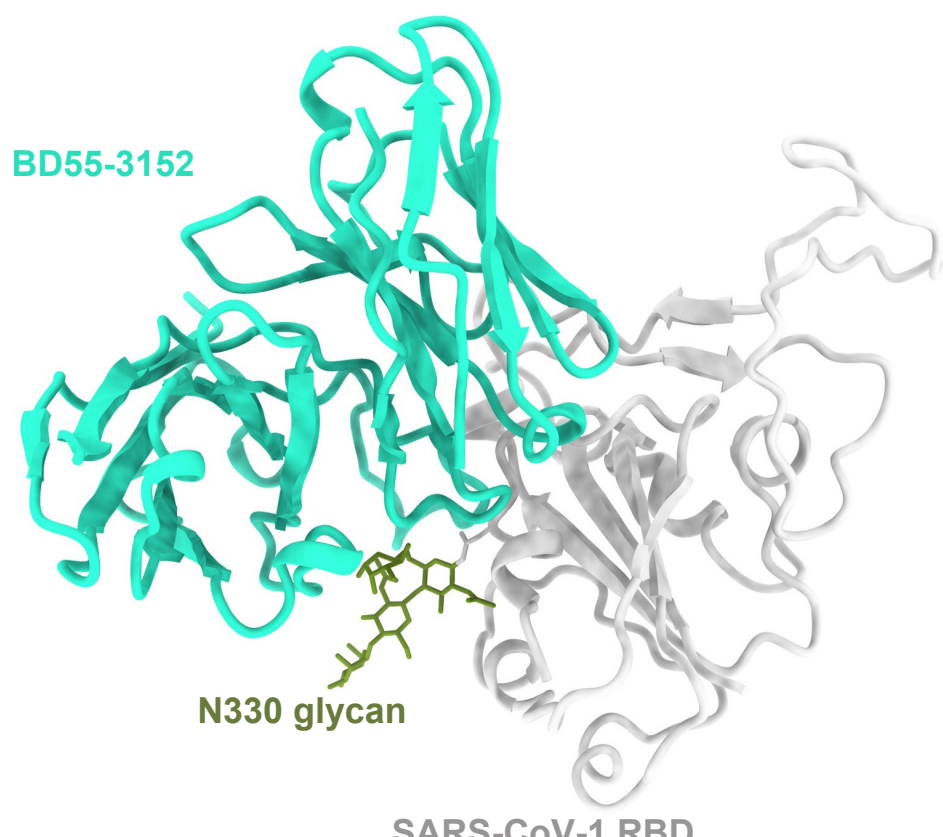

SARS-CoV-1 RBD

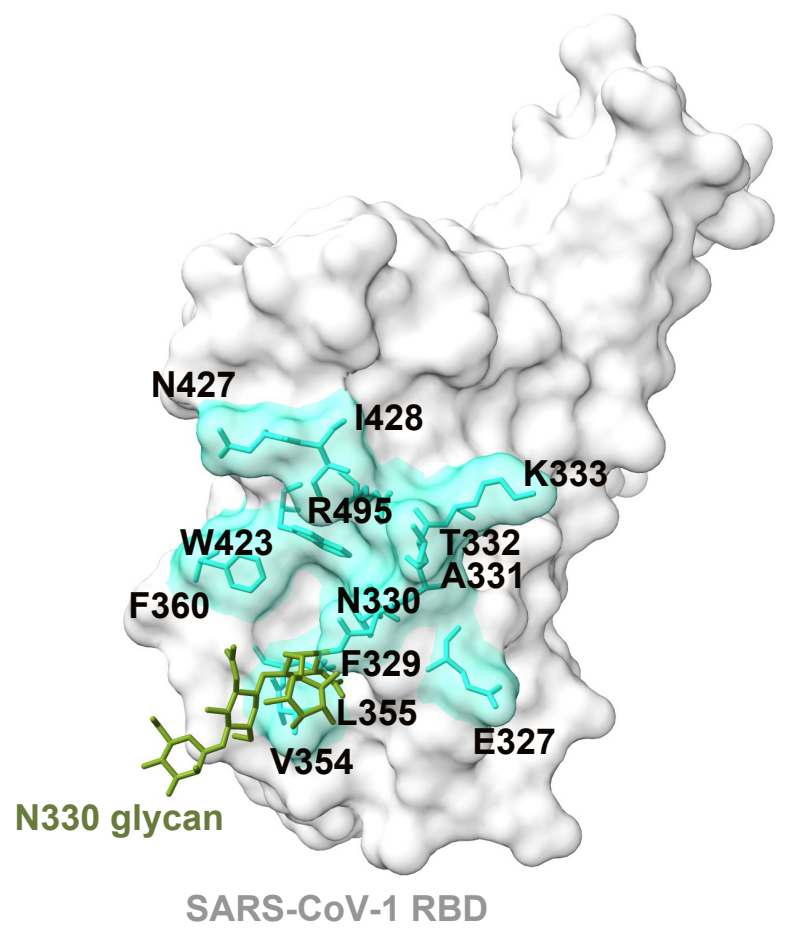

Extended Data Fig. 8 | Cryo-EM structure of BD55-3152 Fab in complex of SARS-CoV-1

RBD.

Residues on the binding interface are marked. 
bioRxiv preprint doi: https://doi.org/10.1101/2022.02.07.479349; this version posted February 7, 2022. The copyright holder for this preprint (which was not certified by peer review) is the author/funder, who has granted bioRxiv a license to display the preprint in perpetuity. It is made available under aCC-BY-NC-ND 4.0 International license.

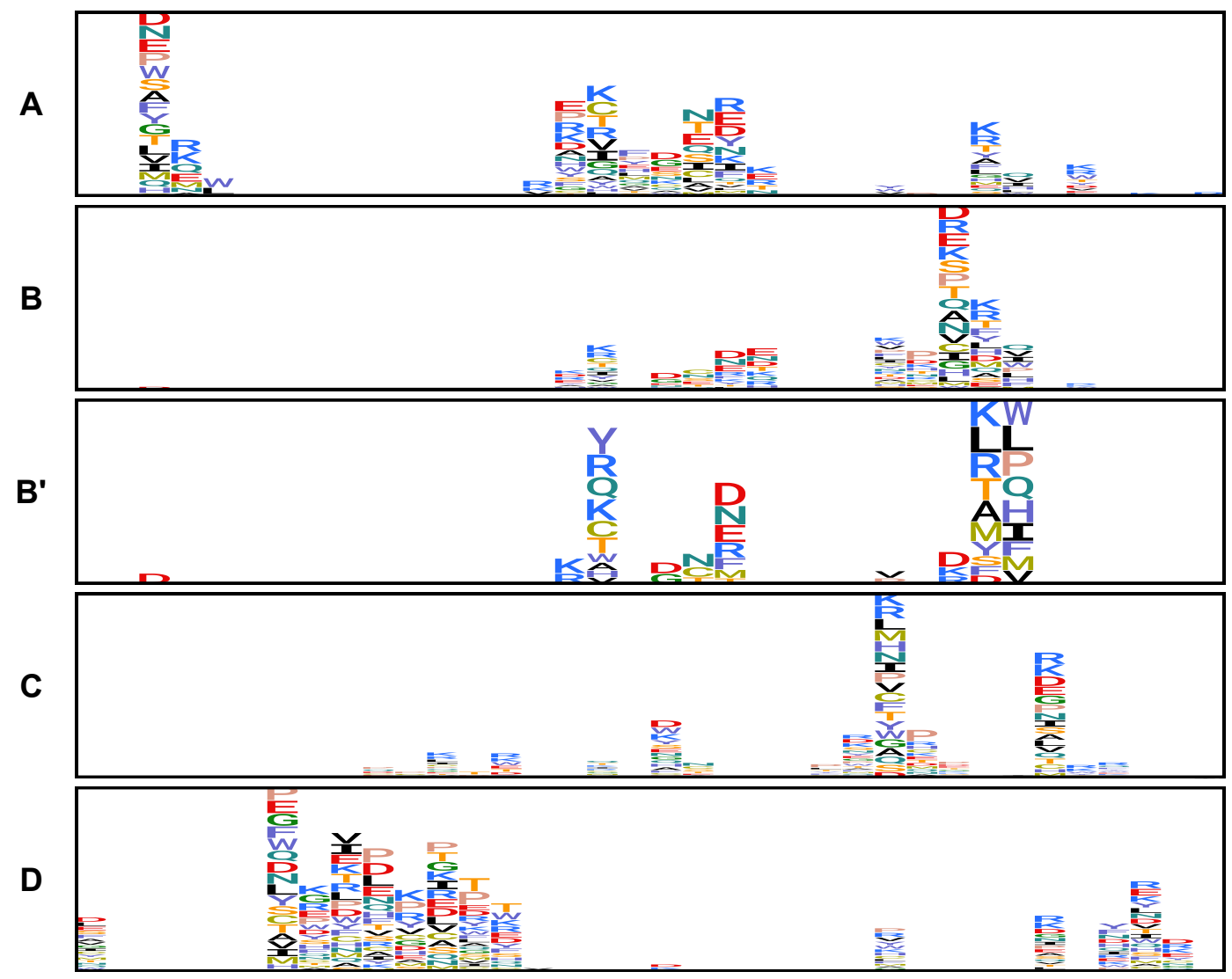

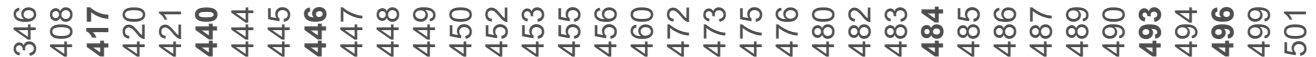

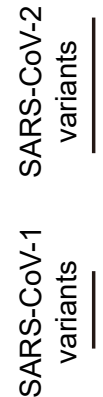

WT R R K D Y N K V G G N Y N L Y L F N I Y A G C G V E G F N Y F Q S GP N

BA.1 R R N D Y K K V S GN Y N L Y L F I Y A GCGVA G F N Y F R S S P Y

BA.1.1 KR N D Y K KV S GNYNL Y L F I Y A G C GVA G F N Y F R S SP Y

BA.2 R S NDYKKV G GN Y N L Y L F N I Y A GCGVA GF N Y F R S GP Y

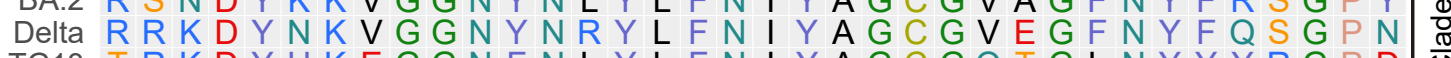

RaTG13 TRKD Y H K E G GN F N L Y L F N I Y A G C GQ T G L N Y Y Y R GP D U

Pangolin-GD TRR D Y N K V G GN Y N L Y L F N I Y A G C G V E GF N Y F Q S GP N Pangolin-GX KR VD Y K L T G GN - - L Y L F K I Y A G C G Q V G L N Y Y E R G P T Urbani KRVDYNTSTGNYNKYYLKPFPDCP-PA L N Y T NDG

Sin852 KRVDYNTSTGNYN KYYLKPFPDCP-PA L N Y WNDG

PC4-127 KRVDYN T S T GNYN K Y Y L K P F P D C P - P A P N YWR G G T S

WIV1 TR VDYNTQTGNYNKYSLKP FPDCP-PAFN YWNDG I N

LYRa11 TR V D Y N T S S G N F K Y S L K P F P D C P - P A F N Y W N D G T $\frac{\pi}{0}$

Rs4231 TR V D Y S S T S GN Y N L Y WV K I Y P G C A - I GP N Y N R P G T A

Rs4084 TR VDYS STS GNYNLYWVK I YP GCA - V GPN Y N R P G T A m

BM48-31 SR VDY S - - S N E - - F Y R F K L F P S C A - E G L N Y K A S G Q S

BtKY72 NR V D Y S K S G N N - - Y Y L F K L Y S A C S I S Q L G Y E K S G V V

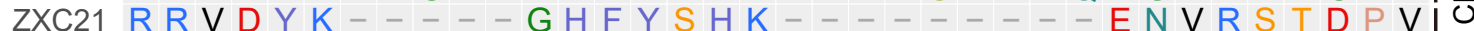

ZC45 RR V D Y K - - - - G N F Y S H K $-\ldots \ldots \ldots$

HeB2013 R R V D Y K $-\ldots \ldots$

Rf4092 R R V D Y K - - - - G S F Y S H K $-\ldots-\ldots$

YN2013 R R V D Y N $\ldots \ldots \ldots$ G S F Y S H K $\ldots \ldots \ldots \ldots$

As6526 R R V D Y Q $\ldots \ldots \ldots$ - $\ldots$ Q Y Y S S K $\ldots \ldots \ldots$

Rs4237 R R V D Y K - - - - GQYYS S K $\ldots \ldots \ldots$

Rp3 R R V D Y K - - - - GQYYSHK $\ldots \ldots \ldots$

Shaanxi2011 R R V D Y N - - - - G Q Y Y S S K $\ldots \ldots-\ldots$

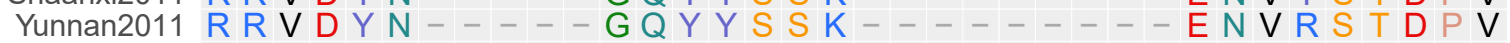

Rs4247 R R V D Y K _ _ $\ldots$ G HYY S H K D $\ldots \ldots \ldots$

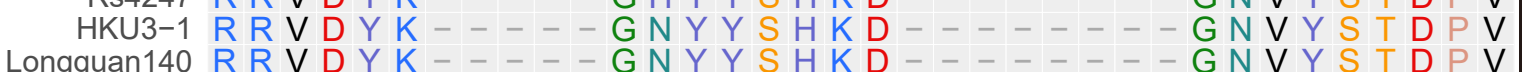

HuB2013 R R V D Y K

G Y Y Y S H K D

G N V Y S T D P V

G N V Y S T D P V

\section{Extended Data Fig. 9 | Averaged escape maps of antibodies in epitope group A-D.}

Height of each amino acid in the escape maps represents its mutation escape score. Residues are colored corresponding to their chemical properties. Mutated sites in Omicron variants are marked in bold. 
bioRxiv preprint doi: $h t t p s: / / d o i . o r g / 10.1101 / 2022.02 .07 .479349 \cdot$ this version posted February 7 2022. The copyright holder for this preprint (which was not certified by peer review) is the author/funder, who has granted bioRxiv a license to display the preprint in

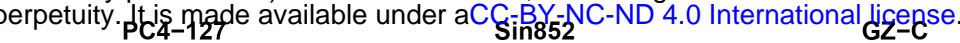

Urbani
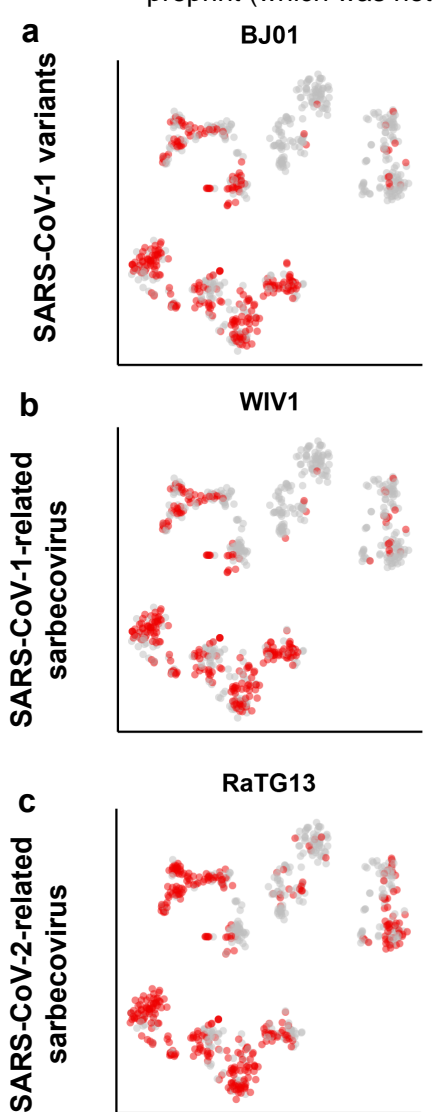

BM48-31

d

e

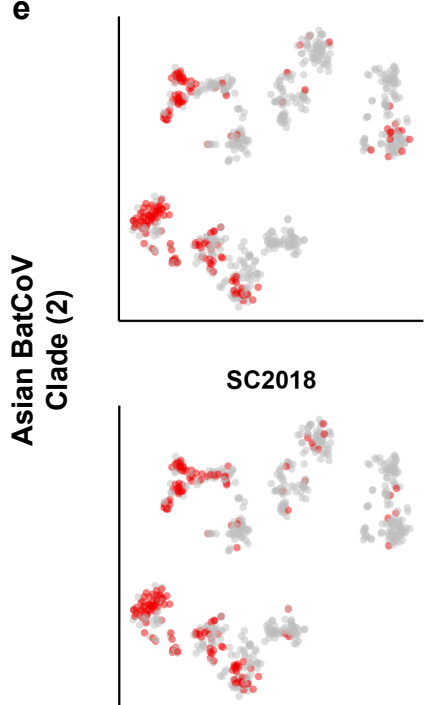

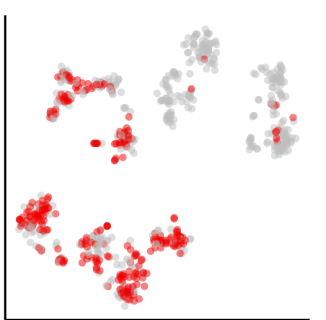

LYRa11

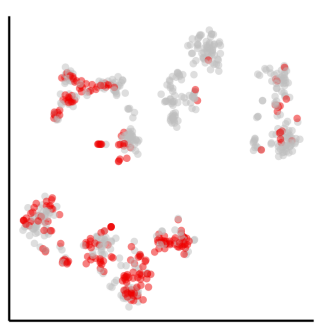

Pangolin-GD

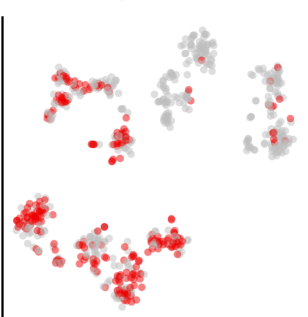

Rs7327

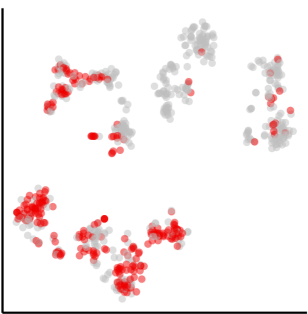

Pangolin-GX

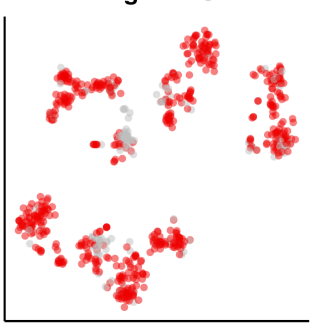

BtKY72

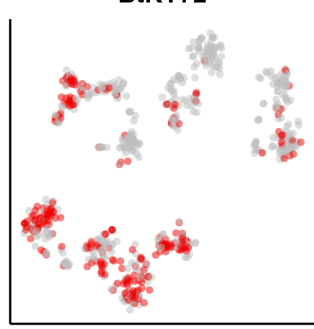

Rs4247

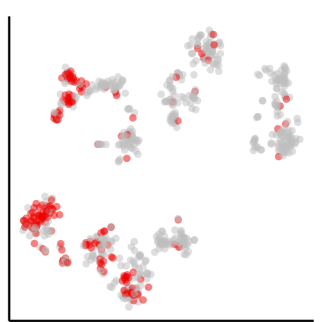

ZC45
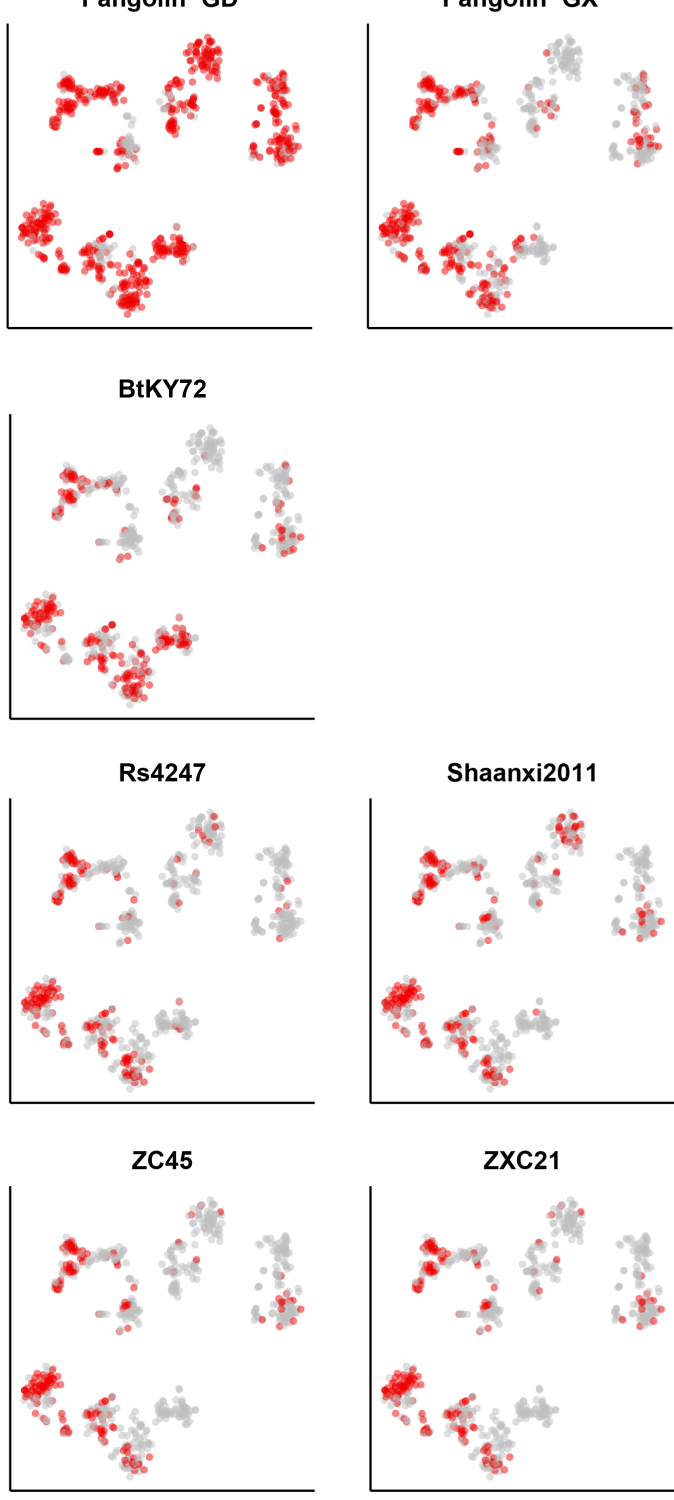

Shaanxi2011

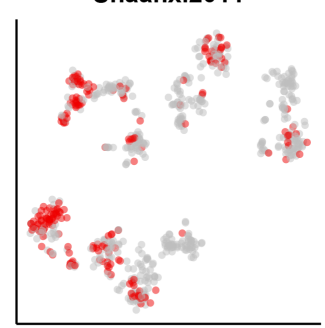

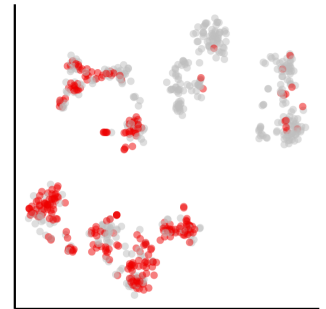

Rs4231
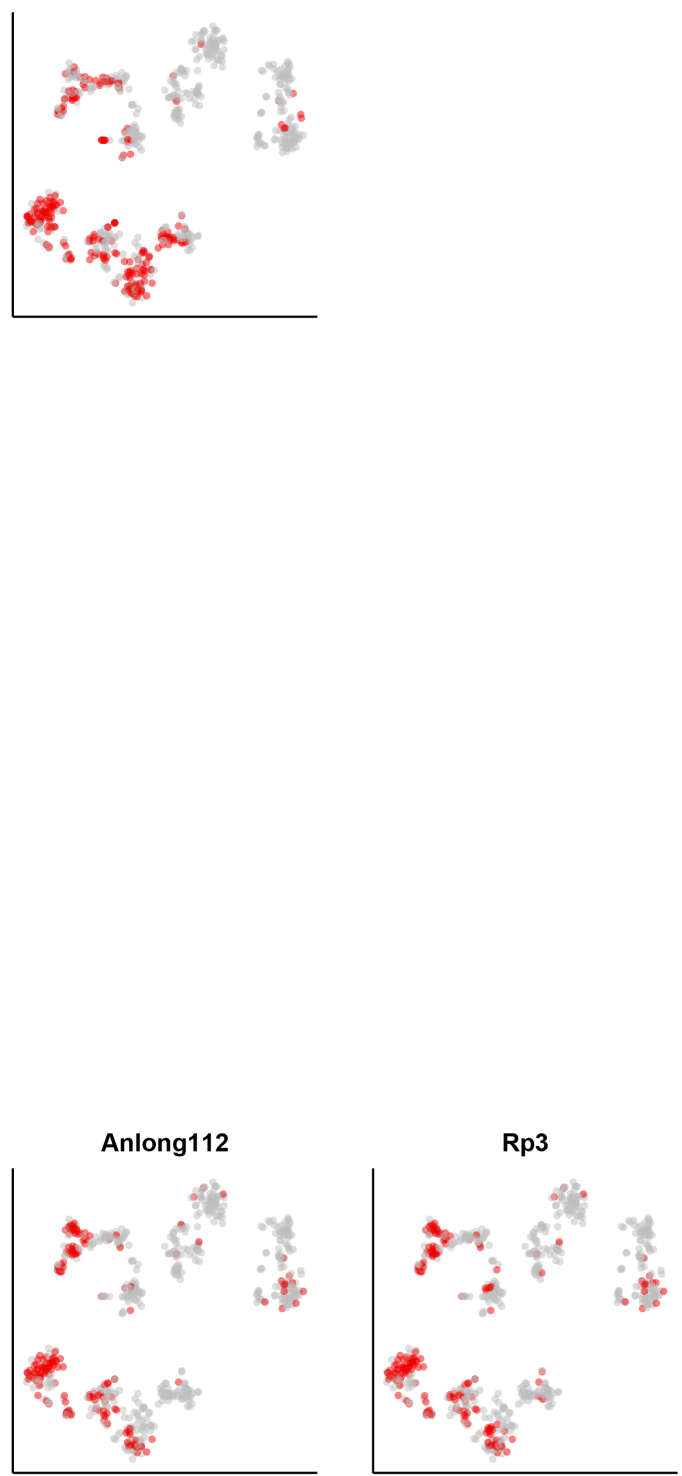

Extended Data Fig. 10 | Projection of DMS predicted escaping of sarbecovirus RBD. a-e, Gray points represent antibodies predicted to be escaped from DMS data. Deletions and insertions on RBD are not considered. (a, SARS-CoV-1 variants. b, SARS-CoV-1-related sarbecovirus. c, SARS-CoV-2 related sarbecovirus. d, Africa/Europe batcoronavirus. e, Asian non-ACE2-utilizing batcoronavirus.) 